\title{
Schwann Cells Generated from Neonatal Skin-Derived Precursors or Neonatal Peripheral Nerve Improve Functional Recovery after Acute Transplantation into the Partially Injured Cervical Spinal Cord of the Rat
}

\author{
DJoseph S. Sparling, ${ }^{1}$ Frederic Bretzner, ${ }^{1}$ Jeff Biernaskie, ${ }^{3}$ Peggy Assinck, ${ }^{1}$ Yuan Jiang, ${ }^{1}$ Hiroki Arisato, ${ }^{1}$ \\ Ward T. Plunet, ${ }^{1}$ Jaimie Borisoff, ${ }^{1}$ Jie Liu, ${ }^{1}$ Freda D. Miller, ${ }^{3,4}$ and Wolfram Tetzlaff ${ }^{1,2}$ \\ ${ }^{1}$ International Collaboration on Repair Discoveries and ${ }^{2}$ Departments of Zoology and Surgery, University of British Columbia, Vancouver, British \\ Columbia, Canada V5Z 1M9, and ${ }^{3}$ Developmental and Stem Cell Biology Group and ${ }^{4}$ Neurosciences and Mental Health, Hospital for Sick Children and \\ University of Toronto, Toronto, Ontario, Canada M5G 1L7
}

The transplantation of Schwann cells (SCs) holds considerable promise as a therapy for spinal cord injury, but the optimal source of these cells and the best timing for intervention remains debatable. Previously, we demonstrated that delayed transplantation of SCs generated from neonatal mouse skin-derived precursors (SKP-SCs) promoted repair and functional recovery in rats with thoracic contusions. Here, we conducted two experiments using neonatal rat cells and an incomplete cervical injury model to examine the efficacy of acute SKP-SC transplantation versus media control (Experiment 1) and versus nerve-derived SC or dermal fibroblast (Fibro) transplantation (Experiment 2). Despite limited graft survival, by 10 weeks after injury, rats that received SCs from either source showed improved functional recovery compared with media- or fibroblast-treated animals. Compared with media treatment, SKP-SC-transplanted rats showed enhanced rubrospinal tract (RST) sparing/plasticity in the gray matter (GM) rostral to injury, particularly in the absence of immunosuppression. The functional benefits of SC transplantations over fibroblast treatment correlated with the enhanced preservation of host tissue, reduced RST atrophy, and/or increased RST sparing/plasticity in the GM. In summary, our results indicate that: (1) early transplantation of neonatal SCs generated from skin or nerve promotes repair and functional recovery after incomplete cervical crush injury; (2) either of these cell types is preferable to Fibros for these purposes; and (3) age-matched SCs from these two sources do not differ in terms of their reparative effects or functional efficacy after transplantation into the injured cervical spinal cord.

Key words: cyclosporine A; forelimb behavior; Schwann cells; skin-derived precusors; spinal cord injury; transplantation

\section{Introduction}

The transplantation of Schwann cells (SCs) has emerged as a promising treatment strategy for spinal cord injury (SCI) (Tetzlaff et al., 2011). Although the majority of preclinical SCI animal studies have used SCs isolated from peripheral nerve (N-SCs), we favor the use of SCs generated from skin-derived precursors

Received March 17, 2014; revised March 18, 2015; accepted March 21, 2015.

Author contributions: J.S.S., F.B., W.T.P., J. Borisoff, F.D.M., and W.T. designed research;J.S.S., F.B., J. Biernaskie, Y.J., H.A., and J.L. performed research; J.S.S., F.B., P.A., Y.J., and H.A. analyzed data; J.S.S. wrote the paper with minor contributions from F.B. and J. Biernaskie and editorial contributions from F.B., J. Biernaskie, P.A., and W.T.

This work was supported by grants from NeuroScience Canada, the Canadian Stem Cell Network, and the Canadian Institutes of Health Research (CIHR). F.B. was funded by Fonds de Recherche en Sante du Quebec and CIHR postdoctoral fellowships. J. Biernaskie was supported by a fellowship from CIHR. P.A. was supported by a CIHR Canadian Graduate Scholarship. We thank Matt Ramer (University of British Columbia) for the use of his electrophysiological equipment; Karim Fouad (University of Alberta) for critical reading of an earlier version of this manuscript; and Clarrie Lam, Fernando Lucero Villegas, Eric Xu, and Tania Morano for technical assistance on various portions of this work. Portions of this work have been published previously in abstract form (Bretzner et al., 2007; Sparling et al., 2009).

The authors declare no competing financial interests.

Correspondence should be addressed to Wolfram Tetzlaff, MD, PhD, Blusson Spinal Cord Centre, 818 West 10th Avenue, Vancouver, BC, Canada V5Z 1M9. E-mail: tetzlaff@icord.org.

DOI:10.1523/JNEUROSCI.1070-14.2015

Copyright $\odot 2015$ the authors $\quad 0270-6474 / 15 / 356714-17 \$ 15.00 / 0$
(SKP-SCs) because skin biopsy is not associated with permanent functional deficits or the risk of chronic pain due to neuroma formation, both of which typically accompany nerve excision (Hood et al., 2009). Similar to N-SCs, SKPs can be isolated from adult tissue (Toma et al., 2001), thereby providing a potential source of autologous SCs for SCI therapy. However, there are also potential sources of neonatal/juvenile SKP-SCs for clinical application because a small skin sample could be collected from virtually any child undergoing pediatric surgery, cryogenically banked, and later used to generate SKP-SCs for autologous or allogeneic transplantation therapies. Therefore, the question of efficacy of neonatal SKP-SCs is clinically relevant despite the fact that the vast majority of SCI patients are adults.

Previously, we demonstrated that neonatal SKP-SCs promote repair and functional recovery after delayed transplantation in rats with thoracic contusions (Biernaskie et al., 2007). Although the transplantation of neonatal SKP-SCs was associated with a variety of reparative effects, these cells failed to induce regeneration of the injured corticospinal tract. This led us to question whether other descending motor tracts might prove more responsive to transplanted SKP-SCs. To address this question, we chose to examine the response of the injured rubrospinal tract 
(RST) to transplanted neonatal rat SKP-SCs using a left cervical (C4/5) dorsolateral funiculus (DLF) crush model of SCI.

Previously, we showed that SKP-SCs exhibited neuroprotective effects when transplanted $7 \mathrm{~d}$ after SCI (Biernaskie et al., 2007), which led us to question whether earlier intervention with these cells might also prove effective. To test this hypothesis, we used immediate transplantation of SKP-SCs here to establish proof of principle regarding the utility of these cells as an acute SCI therapy and conducted two independent experiments using that injury/transplantation paradigm. Experiment 1 aimed to establish efficacy by comparing neonatal SKP-SC therapy with media treatment and examined the effect of immunosuppression with cyclosporine A (CsA). Experiment 2 compared the efficacy of acutely transplanted neonatal SKP-SCs with age-matched $\mathrm{N}$-SCs and dermal fibroblasts (Fibros). The use of neonatal SKPSCs and N-SCs allowed us to test whether phenotypic differences exist between age-matched SCs from these two sources, whereas Fibros provided a cellular control that comes from the same source tissue as SKPs (i.e., mammalian dermis). This work represents the first assessment of SKP-SCs as a treatment for cervical SCI and as an early intervention for SCI, as well as of their influence on the injured RST and their response to immunosuppression with CsA, and is also the first side-by-side comparison of the efficacy of SKP-SCs and N-SCs as SCI therapies.

\section{Materials and Methods}

\section{Procedures}

All experimental procedures used in this work were approved by the Animal Care Committee of the University of British Columbia and/or the Hospital for Sick Children Research Institute in accordance with the guidelines of the Canadian Council on Animal Care. Two separate experiments were conducted: the first (Experiment 1) compared SKP-SC transplantation with media treatment alone and the second (Experiment 2) compared the effects of transplanting SKP-SCs, N-SCs, or Fibros. It should be noted that the two experiments reported here were conducted many months apart and by different lead researchers (J.S.S. and F.B.) and, despite the fact that the same methods, equipment, and personnel were largely used in both studies, the results in Experiments 1 and 2 differed significantly on a number of outcome measures. In light of that, we chose to present and analyze the two experiments independently throughout this work.

\section{Animals and experimental design}

For ease of tracking transplanted cells and their progeny, we generated all of the cells used in this study from transgenic Sprague Dawley (SD) rats expressing GFP (National Bio Resource Project, Kyoto, Japan). In light of the higher clinical prevalence of SCI in males (Wyndaele and Wyndaele, 2006) and because sex may influence spontaneous repair in adult rodents (Li et al., 2006), we chose to use only male rats as transplant recipients. A total of 97 adult male SD rats $(300-500 \mathrm{~g})$ were used in this work, 10 of which were killed before the end point due to surgical/health complications. These complications were primarily related to autotomy of the digits on the hindlimb contralateral to the cervical injury, which is a common complication of the present injury model. Twenty-two wildtype SD rats (Charles River) served as uninjured controls for behavioral, electrophysiological, and/or axonal tracing analyses. To minimize animal use, both GFP-negative $\left(\mathrm{GFP}^{-}\right)$SD rats (UBC Animal Care Facility, Vancouver, BC) and wild-type (WT) SD rats (Charles River) were used in Experiment 1. Given that SD is an outbred rat strain and that the $\mathrm{GFP}^{-}$ rats were all six or more generations removed from the animals used to generate the cells for transplantation in this study, all transplants in this study should be considered allogeneic. Thirty injured/treated rats reached the designated end point (media: $n=10$; SKP-SC: $n=20$ ) in Experiment 1. All of the animals used in Experiment 2 were WT SD rats (Charles River) and, of the rats that were injured and treated in this study, 35 (SKP-SC: $n=11$; N-SC: $n=13$; Fibro: $n=11$ ) reached the end point. Half of the animals in each treatment group in Experiment 1 and all of the animals in Experiment 2 were immunosuppressed with CsA (Novartis Pharmaceuticals) to prevent $\mathrm{T}$-cell-mediated immune rejection. CsA was administered via daily injection ( $10 \mathrm{mg} / \mathrm{kg} / \mathrm{d}$, i.p.) from $2 \mathrm{~d}$ before surgery until 2 weeks after surgery, after which point oral CsA was provided in home cage drinking water (Neoral; Novartis; $1.5 \mathrm{ml} / \mathrm{L}$ of water) for the duration of the experiments.

\section{Cell culture for transplantation}

Three different cell types (SKP-SCs, N-SCs, and Fibros) were generated from GFP-positive $\left(\mathrm{GFP}^{+}\right)$neonatal SD rat tissue for transplantation into the injured DLF in this study. The isolation of SKPs and differentiation of SKP-SCs followed the same methods in both experiments. In Experiment 2, SKPs, N-SCs, and Fibros were all isolated from the same pups. All cells were passaged 3-4 times before transplantation, thereby ensuring purity via sequential passaging under cell-specific growth conditions. Using the methods outlined below, our cultures of these three cell types all typically display purity $\geq 95 \%$ according to estimates based on morphology and/or labeling with typical SC markers (e.g., p75 and S100) and it should be noted that each of the SC cultures used in the present work demonstrated densely packed, parallel, swirling arrays of bipolar cells (characteristic of highly pure cultures of SCs) before transplantation.

Isolation of SKPs and differentiation of SKP-SCs. SKPs were isolated, expanded, and differentiated into high-purity SKP-SCs as described previously (Biernaskie et al., 2006; Biernaskie et al., 2007).

Isolation of N-SCs. N-SCs were isolated from neonatal sciatic nerve segments. After dissection, the nerve segments were minced and digested in collagenase type $\mathrm{XI}$ at $37^{\circ} \mathrm{C}$ for $30-60 \mathrm{~min}$. Cells were liberated by gentle trituration and the dissociated cells were plated on $10 \mathrm{~cm}$ dishes coated with laminin/PDL and grown in DMEM:F12 (3:1) containing 50 $\mathrm{ng} / \mathrm{ml}$ neuregulin- $1 \beta, 4 \mu \mathrm{g} / \mathrm{ml}$ insulin (Invitrogen), $5 \mu \mathrm{M}$ forskolin, $2 \%$ $\mathrm{N} 2$ supplement, and, in some cases, $1 \%$ FBS.

Isolation of Fibros. Fibros were isolated from neonatal glabrous (i.e., nonhairy) skin from the ventral surface of the hindfeet. Epidermis was removed after a $15 \mathrm{~min}$ incubation in trypsin-EDTA (0.25\%; Invitrogen) and the isolated dermis was then digested in collagenase type XI for $30-60 \mathrm{~min}$ at $37^{\circ} \mathrm{C}$. Cells were liberated by gentle mechanical dissociation and grown adherently on $10 \mathrm{~cm}$ dishes in DMEM:F12 (3:1) containing 5\% FBS, $40 \mathrm{ng} / \mathrm{ml} \mathrm{bFGF}$, and 1\% B27 supplement.

\section{SCI and cell transplantation}

DLF crush. Rats were anesthetized with a mixture of ketamine hydrochloride $(70 \mathrm{mg} / \mathrm{kg}$, i.p.; Bimeda-MTC) and xylazine hydrochloride (10 $\mathrm{mg} / \mathrm{kg}$ i.p.; Bayer). A laminectomy was performed on the left side, exposing the fourth and fifth cervical segments $(\mathrm{C} 4 / 5)$, and the dura was cut with microscissors to expose the spinal cord on the left side. A pair of custom-designed fine surgical forceps (marked at a distance of $1 \mathrm{~mm}$ from the tip; image provided in Hilton et al., 2013) were carefully inserted around the left DLF (containing the RST), lowered to a depth of 1 $\mathrm{mm}$, and completely closed to crush the DLF for $20 \mathrm{~s}$, as described previously (Ramer et al., 2004b; Richter et al., 2005).

Cell transplantation. Rather than conducting a separate transplantation surgery only h after injury, we chose to minimize animal stress and discomfort by transplanting cells immediately after DLF crush injury. SKP-SCs (Experiments. 1 and 2), N-SCs (Experiment 2), or Fibros (Experiment 2) were transplanted into the spinal cord using procedures modified from our previous work (Bretzner et al., 2008, 2010). Briefly, adherent cells were detached from the dish by digestion in trypsin-EDTA $(0.01 \%)$, the trypsin was deactivated by adding $10 \% \mathrm{FBS}$, and the cells were spun down and resuspended in fresh HBSS (Invitrogen; Experiment 1) or DMEM (Experiment 2) at $\sim 135,000$ cells/ $\mu$ l. Resuspended cells were drawn into a pulled glass pipette (with a diameter of $60-80$ $\mu \mathrm{m}$ ) fitted to a $10 \mu \mathrm{l}$ Hamilton microsyringe. Three stereotaxic microinjections of $0.5 \mu \mathrm{l}$ of cell suspension were applied to the crush site, 2 at the medial edge (at 0.5 and $1 \mathrm{~mm}$ depths) and 1 at the lateral edge ( 0.5 $\mathrm{mm}$ depth) of the lesion, for a total of $1.5 \mu$ l containing 200,000 cells. Media control animals in Experiment 1 received equivalent injections of the same volume of HBSS without cells. The glass pipette was left in place for $5 \mathrm{~min}$ after each injection to ensure that cells remained in the spinal 
cord and were not withdrawn with the syringe. After the final injection, the muscle and skin were repositioned and sutured to close the wound. Rats received Ringer's solution $(5 \mathrm{ml}$, s.c.) to maintain hydration and buprenorphine $(0.3 \mathrm{mg} / \mathrm{kg})$ to alleviate postsurgical pain. Rats that reopened their surgical wounds or developed new wounds were treated with antibiotics (Baytril, $0.06 \mathrm{ml}$, i.m.) to prevent infection.

\section{Behavioral testing}

In Experiment 1, behavioral testing using the cylinder test was used to assess recovery of forelimb motor function at 6 and 10 weeks postinjury (wpi) (see Fig. 1A). Logistical complications prevented baseline testing of the animals before injury, so an uninjured control group was assessed to provide a proxy for preinjury behavior. In Experiment 2, cylinder and CatWalk (Noldus) data were collected before injury and again at 6,8 , and 10 wpi (Fig. 1B); preinjury data collection eliminated the need for an uninjured behavioral control group. In both experiments, electrophysiological measures and axon tracing procedures were conducted within days of the 10 wpi behavioral assessments and precluded additional behavioral testing. All behavioral data were scored by raters blinded to the experimental treatment given to each animal.

Cylinder test. The use of each forelimb during vertical exploration was assessed by videotaping animals' rearing behavior in a closed Plexiglas cylinder $(20 \mathrm{~cm}$ in diameter $\times 30 \mathrm{~cm}$ high; Liu et al., 1999). Forelimb use was scored by counting the number of times an animal contacted the wall of the cylinder using the left forelimb only (affected by injury), the right forelimb only (unaffected by injury), or both forelimbs simultaneously during rearing. To accurately reflect the overall use of the forelimb affected by injury, we analyzed these data as the percent use of the "left plus both" forelimbs relative to the total number of forelimb contacts.

CatWalk. As a measure of locomotor function, we used the CatWalk device to analyze a wide variety of forelimb and hindlimb gait parameters (Hamers et al., 2001, 2006). This device captures the shape, size, orientation, and timing of each paw placement and provides a measure of the pressure each paw exerts on the glass (i.e., paw intensity). The animals were trained to cross the walkway in a darkened room and multiple crossings were recorded for each animal at each time point. Using CatWalk version 7.1 software, we averaged values from five steady runs with a minimum of three complete step cycles at every time point to generate data on a wide variety of parameters (see Results) for each animal at each time point and these values were used to generate group averages for subsequent statistical analyses.

\section{Electrophysiology}

We assessed the synaptic efficacy of the injured RST by recording electromyographic (EMG) responses in the left forelimb (affected by injury) evoked by electrical stimulation of the contralateral red nucleus. These assessments were conducted on uninjured control rats and on all injured/ treated rats in both experiments. Rats were anesthetized with a mixture of ketamine and xylazine (as described above) and placed in a stereotaxic frame. Anesthesia was maintained by administering additional doses of ketamine as needed throughout the procedure. EMG responses were recorded via bipolar electrodes made of insulated copper microwires with exposed tips of 2-3 mm inserted into the left forearm (extensor digitorum communis and/or extensor carpi radialis) muscles. A tungsten electrode was then lowered into the red nucleus at a depth of $7 \mathrm{~mm}$ below the dura through an opening in the skull at $6.1 \mathrm{~mm}$ posterior to bregma and $1.7 \mathrm{~mm}$ lateral (right) of the midline. The optimal stimulation site was located by varying the depth of the electrode during test stimulations until motor and EMG responses were elicited in the forearm muscles. EMG responses were evoked at that depth using the lowest current strength possible and the stimulus intensity was recorded as the motor threshold for each animal. Animals that failed to provide EMG responses at coordinates within $1 \mathrm{~mm}$ of the average depth of the red nucleus were excluded from this analysis. A short train of 11 cathodal stimuli was applied at a frequency of $330 \mathrm{~Hz}$, with a pulse duration of $0.2 \mathrm{~ms}$ to evoke motor and EMG responses. EMGs were recorded using a Pentusa neurophysiology workstation (Tucker-Davis Technologies), digitized at a sampling rate of $5 \mathrm{kHz}$, and band-pass filtered between 5 and $500 \mathrm{~Hz}$ to eliminate background noise. The latency of evoked EMG responses was measured with respect to the onset of stimulation at the motor threshold and averaged from at least $15 \mathrm{EMG}$ traces per animal.

\section{Anterograde labeling of RST axons}

Immediately after the completion of the electrophysiological measures, anterograde axonal tracing was conducted by stereotaxically injecting biotinylated dextran amine (BDA; $0.6 \mu \mathrm{l}, 10 \mathrm{kDa}, 25 \%$ in $0.5 \%$ dimethylsulfoxide; Invitrogen) into the red nucleus on the right side of the brain at a rate of $80 \mathrm{nl} / \mathrm{min}$ via a pulled glass pipette (diameter of $20 \mu \mathrm{m}$ ) fitted to a Hamilton microsyringe. The coordinates used for these injections were the same as those used for red nucleus stimulation (see above), but the depth of the injection was kept constant at $7 \mathrm{~mm}$ below the dura. The pipette remained in place for $5 \mathrm{~min}$ after injection to ensure that BDA was not withdrawn with the syringe. Six uninjured control animals were also subjected to BDA tracing, concurrent with the tracing of animals in Experiment 2, to establish baseline levels for RST axon density measures.

\section{Tissue processing and immunohistochemistry}

Eleven weeks after injury and treatment (i.e., 1 week after electrophysiology and tracing procedures), rats were anesthetized with a lethal dose of chloral hydrate $(100 \mathrm{mg} / \mathrm{kg}$, i.p.; BDH Chemicals) and perfused transcardially with PBS followed by phosphate buffered, $4 \%$ paraformaldehyde, $\mathrm{pH}$ 7.4. Cervical segments of spinal cord were dissected, postfixed in $4 \%$ paraformaldehyde overnight, cryoprotected in $24 \%$ sucrose in $0.1 \mathrm{M}$ phosphate buffer over 2-3 d, and frozen in Tissue-Tek (Fisher Scientific) using isopentane chilled with dry ice. Spinal cords were cut into $20 \mu \mathrm{m}$ sections on a cryostat, mounted on Superfrost Plus slides (Fisher Scientific), and stored at $-80^{\circ} \mathrm{C}$. The cervical spinal cord from C3 to C6 was sectioned longitudinally in the horizontal plane, whereas segments from C2 (above the lesion) were sectioned in the coronal plane. For immunohistochemistry, frozen sections were thawed at room temperature for 30-60 min, rehydrated in $0.01 \mathrm{M}$ PBS for at least $10 \mathrm{~min}$, and then incubated with $10 \%$ normal donkey serum and/or $10 \%$ normal goat serum containing $0.1 \%$ Triton X-100 (Sigma-Aldrich) for $30 \mathrm{~min}$ to block nonspecific binding. Sections being immunolabeled for myelin protein zero $\left(\mathrm{P}_{0}\right)$ underwent alcohol delipidation to increase epitope availability before serum block. Primary antibodies were diluted in 0.01 м PBS containing $0.1 \%$ Triton X-100 and incubated on tissue overnight at room temperature or $4^{\circ} \mathrm{C}$. After primary antibody incubation, the tissue was washed and secondary antibodies (diluted in $0.01 \mathrm{~m}$ PBS alone) were applied for $2-3 \mathrm{~h}$ at room temperature, followed by multiple washes and coverslip application. The following primary antibodies were used: chicken anti-GFP (1:1000; Millipore Bioscience Research Reagents), rabbit anti-GFP (1:1000; Millipore Bioscience Research Reagents), goat anti-GFP (1:400; Rockland Immunochemicals), mouse anti-glial fibrillary acidic protein (GFAP; 1:400; Sigma-Aldrich), rabbit anti-GFAP (1: 1000; Dako), goat anti-GFAP (1:50; Santa Cruz Biotechnology), mouse anti-neurocan (1:100; Developmental Studies Hybridoma Bank), mouse anti-neurofilament 200 (NF; 1:500; Sigma-Aldrich), mouse anti- $\beta$ IIItubulin ( $\beta$ IIItub; 1:400; Sigma-Aldrich), rabbit anti-serotonin transporter (SERT; 1:500; Sigma-Aldrich), sheep anti-tyrosine hydroxylase (TH; 1:200; Millipore Bioscience Research Reagents), chicken antiprotein zero $\left(\mathrm{P}_{0} ; 1: 100\right.$; Aves Labs), mouse anti-rat p75 neurotrophin receptor ( $\mathrm{p} 75$; 1:500; Millipore Bioscience Research Reagents), rabbit anti-p75 (1:400; Millipore Bioscience Research Reagents), rabbit antilaminin (1:200; Sigma-Aldrich), mouse anti-fibronectin (1:200; Dako), and rabbit anti-collagen type I (Collagen; 1:60; Millipore Bioscience Research Reagents). Secondary antibodies included: FITC-, Cy3-, and AMCA-conjugated donkey anti-chicken, mouse, or rabbit antibodies (all 1:200; Invitrogen); Alexa Fluor 488-conjugated goat anti-chicken (1:200; Invitrogen); donkey anti-chicken, mouse, rabbit, goat, or sheep antibodies conjugated to Dylight 405 (1:200), Dylight 488 (1:400), Dylight 594 (1:200), or Dylight 649 (1:300) (all Jackson ImmunoResearch). BDA was visualized by using Cy3- or AMCA-conjugated streptavidin (1:400; Jackson ImmunoResearch) applied for the duration of secondary antibody incubation. In some sections, we also stained nuclei with Hoechst 33342 (1:5000; Invitrogen), which was applied for 5 min after secondary antibody incubation. 


\section{Image analysis and histological quantifications}

Image acquisition and processing. Immunofluorescence was captured digitally using an Axioplan 2 microscope (Zeiss) equipped with Northern Eclipse software (Empix Imaging) or a Zeiss AxioObserver Z1 confocal microscope fitted with a CSU-X1 spinning disc (Yokogawa Electric) and AxioVision 4.8 (Zeiss). Digital images were processed for presentation and quantification using Photoshop 7.0 or 9.0 (Adobe Systems); SigmaScan Pro 5 (SPSS), or MATLAB 6.5 (The MathWorks) software. Rostral is up and caudal is down in all of the sample images in every figure in this work, except Figures 4, $\mathrm{N}$ and $\mathrm{O}$, and $5 \mathrm{H}$, where rostral is right and caudal is left. All histological analyses were conducted by individuals blinded to the treatment of each animal.

Area, volume, and distance quantifications. Lesion, cavity, and GFP ${ }^{+}$ graft areas were quantified every $320 \mu \mathrm{m}$ through the extent of the injury site by outlining the GFAP-negative $\left(\mathrm{GFAP}^{-}\right)$area, the area devoid of cytoarchitecture, or the $\mathrm{GFP}^{+}$area within the spinal cord, respectively. The total number of pixels within each of these areas was measured using SigmaScan Pro5 and the total lesion, cavity, and $\mathrm{GFP}^{+}$graft volume were estimated for each animal using the following formula: volume $=\Sigma$ (area $\times$ section thickness $\times$ number of sections between samples). Using the same approach, we estimated the $\mathrm{GFAP}^{+} / \mathrm{GFP}^{+}$volume for each animal and used that to calculate the percentage of the lesion occupied by the $\mathrm{GFP}^{+}$graft for each animal. To examine the integration of transplanted SCs with GFAP ${ }^{+}$astrocyte-rich host parenchyma, we measured the total GFP ${ }^{+}$pixel area found in regions of $\mathrm{GFAP}^{+}$intact parenchyma outside of the lesion (using the sampling strategy outlined above), and then averaged these measures of SC-astrocyte overlap to get an individual "integration area" for each animal in the SKP-SC and N-SC groups in Experiment 2. For a subset of six animals randomly selected from each group, we used the sampling strategy described above to measure the $\mathrm{P}_{0}$-positive $\left(\mathrm{P}_{0}{ }^{+}\right)$and $\mathrm{GFP}^{+}$areas, as well as the area of overlap (both $\mathrm{P}_{0}{ }^{+}$and $\left.\mathrm{GFP}^{+}\right)$. Importantly, we thresholded images to eliminate any background signal to ensure that we were only measuring pixels associated with $\mathrm{GFP}^{+}$and $\mathrm{P}_{0}{ }^{+}$structures before assessing overlap and were careful to ignore artifacts that did not have appropriate morphology. These area values were used to calculate the percentage of $\mathrm{GFP}^{+}$area that was also $\mathrm{P}_{0}{ }^{+}$(an estimate of the SC myelin produced by transplanted cells and their progeny) and the percentage of $\mathrm{P}_{0}{ }^{+}$area that was GFP ${ }^{-}$ (an estimate of the SC myelin produced by endogenous SCs). The in vivo migration potential of transplanted SKP-SCs and N-SCs was assessed by measuring the shortest possible distance between the edge of the lesion site and the single farthest SC from the lesion in each of the tissue sections used for the aforementioned integration area analysis. Cells that had clearly migrated outside of the cord or through the central canal were ignored and the maximum migration distance for a single SC from each animal was used in the final statistical analysis. Spared rim width was assessed by measuring the thinnest width of the intact/continuous $\mathrm{GFAP}^{+}$tissue separating the outside edge of the spinal cord from the inside edge of the lesion on two longitudinal GFAP-labeled sections (320 $\mu \mathrm{m}$ apart) near the lesion epicenter. These two values were averaged to generate the spared rim width for each animal in subsequent statistical analysis.

Immunoreactivity quantifications. GFAP immunoreactivity (GFAPIR) and neurocan-IR were evaluated using SigmaScan Pro5 to measure the average fluorescence intensity within a $50-\mu \mathrm{m}$-wide line circumscribing the entire rostral or caudal edge of the lesion site (for overall GFAP-IR and neurocan-IR; all groups) or through segments of that region immediately adjacent to $\mathrm{GFP}^{+}$cells (GFAP-IR near transplantderived SCs; Experiment 2 SKP-SC and N-SC groups only) on three longitudinal sections spaced $320 \mu \mathrm{m}$ apart in each animal. Image settings were chosen to avoid oversaturation of pixels adjacent to the lesion and care was taken to avoid artifacts and the very edge of the spinal cord. The measurement line drawn on a given section was also used to measure the intensity of an analogous area on the uninjured side of the spinal cord and these control values were used to normalize the intensity measurements in each section to control for potential differences in immunoreactive labeling across different tissue sections. Normalized values from each section were averaged to attain mean GFAP-IR and neurocan-IR scores rostral and caudal to injury for each animal. To ensure that the intensity of neurocan labeling in the gray matter (GM; where neurocan expression is much higher) did not mask group differences in the white matter (WM) rostral or caudal to injury, we repeated the neurocan analysis measuring intensity in WM regions only. There were no significant differences in neurocan-IR among the groups using either method, so we present the data from the WM analysis here in Figure 5.

GM and WM RST axon quantifications. The level at which the RST branches in the GM was located by examining longitudinal tissue sections (every $20 \mu \mathrm{m}$ in $180 \mu \mathrm{m}$ blocks spaced $140 \mu \mathrm{m}$ apart) between the DLF and the central canal to find the highest concentration of BDApositive $\left(\mathrm{BDA}^{+}\right) \mathrm{RST}$ axons branching in each animal. We measured axon density in the GM at that level in three longitudinal sections spaced $60 \mu \mathrm{m}$ apart and in three coronal sections spaced $60 \mu \mathrm{m}$ apart at C2. $\mathrm{BDA}^{+} \mathrm{RST}$ axons within the GM $1 \mathrm{~mm}$ rostral ("far rostral"), immediately rostral ("rostral"), and immediately caudal ("caudal") to the injury site at C4/5 were imaged from each longitudinal section and the left DLF and adjacent GM were imaged on each coronal section. The RST axon density measurement used here was adapted from Ramer et al. (2004a). In brief, threshold overlays were created for each of these images, in which all pixels associated with $\mathrm{BDA}^{+}$axons were assigned a grayscale value of 68 and all other pixels were assigned a value of 0 , and GM RST axon density values were generated by dividing the average intensity of a given region of each overlay by 68 . An average of three measures at each level provided the final raw RST axon density values in the GM at C2, far rostral, rostral, and caudal levels of analysis for each animal. In addition, we counted BDA ${ }^{+}$RST axon profiles in the WM at C2 ("axon counts") to measure of the number of descending RST axons in the DLF that successfully transported BDA. Three animals with axon counts $<100$ were excluded from analysis entirely due to failure of $\mathrm{BDA}$ tracing and five animals with damage to the DLF in C2 coronal sections were excluded from the axon count analysis, leaving 54 and 59 animals in the final analyses of axon counts and axon density, respectively.

We originally planned to normalize all of the raw RST axon density data to the axon counts in both experiments, as is commonly done to control for random variability in BDA tracing efficacy from animal to animal. However, we opted not to do so when we found that $\mathrm{BDA}^{+}$axon counts differed significantly among the groups in Experiment 2 (see Fig. $6 \mathrm{H}$ and Results). All of the animals in Experiment 2 were traced in random order using the same batch of BDA injected by the same individual who was blinded to treatment, so the differences among the groups cannot be accounted for by potential differences in tracing procedures/reagents and therefore likely represent meaningful treatment effects (see Discussion). In light of that, we decided that it was inappropriate to normalize the RST axon density data to the axon counts in Experiment 2 because doing so would not only control for random variability in $\mathrm{BDA}$ tracing efficacy, but would also eliminate any variability arising from treatment-related group differences in axon counts. Rather than normalizing data from one experiment but not the other, we opted to present and analyze the raw data from both experiments (see Fig. 6). It is interesting that subsequent analysis of the normalized data from Experiment 1 (data not shown) demonstrated the same overall pattern of statistical results compared with the raw data from that experiment, which indicates that random variability in BDA tracing efficacy did not change the outcome of that analysis.

\section{Statistical analysis and data presentation}

All statistical analyses presented herein were conducted using SPSS 19/20 software; all graphical representations of data were made using GraphPad Prism 5 and processed using Photoshop CS2 (Adobe). All behavioral data are presented as mean \pm SEM and were analyzed using independent-samples $t$ tests (Experiment 1) or repeated-measures ANOVA (RM-ANOVA; Experiment 2), as appropriate. Significant RM-ANOVA effects were followed up with one-way ANOVAs and conservative post hoc testing (Bonferroni) at individual time points as appropriate. All of the nonbehavioral datasets in this study had at least one group that violated the assumptions of normality and/or homogeneity of 
variance that must be met to conduct parametric analyses, so all electrophysiological and histological analyses were conducted using nonparametric tests and are presented as individual data points with group medians indicated by black lines on the graphs. For independent tests involving two or more groups (e.g., comparisons among the treatment groups in Experiment 2), we used the Kruskal-Wallis ranked sum test $\left(\chi^{2}\right)$, followed by pairwise testing with the Mann-Whitney $U$ test. The latter test was also used to compare each injured/treated group in both experiments with the uninjured control group as appropriate. Correlational analyses were conducted using parametric (Pearson's correlation coefficient $[r]$ ) or nonparametric (Spearman's rank correlation coefficient $[\rho]$ ) tests as appropriate for the particular variables and groups included in each analysis. Two-tailed test results are reported for all analyses and, where appropriate, the degrees of freedom for statistical tests are provided in brackets. The significance level for all statistical tests was set at $p<$ 0.05 , but to counteract inflation in the rate of type I errors due to multiple comparisons, we manually applied a Bonferroni correction to all follow-up pairwise tests by dividing $\alpha(0.05)$ by the number of pairwise comparisons being conducted on a given dataset. For example, $\alpha$ was adjusted to $0.05 / 6=0.0083$ when all of the possible pairwise comparisons were made for the groups from Experiment 2 (SKP-SC, N-SC, Fibro, and uninjured) on the RST axon density analysis. The adjusted significance levels used in each analysis are indicated in the figure legends and results where appropriate. Although our use of nonparametric tests and the Bonferroni correction provide for quite conservative statistical analyses, we did not apply a correction for the overall number of tests conducted across different datasets, so our approach does not guarantee that there were no type I errors reported in the present work.

\section{Results}

Here, we report the results of two experiments, the first assessing the efficacy of SKP-SCs as an acute treatment for incomplete cervical SCI and the second comparing SKP-SCs with SCs generated from N-SCs or Fibros in the same injury/transplantation paradigm (for details and timelines, see Fig. 1). In addition to testing different treatments, these experiments differed with respect to immunosuppression because only half of the animals in Experiment 1 received CsA compared with all of the animals in Experiment 2. Preliminary testing revealed that, regardless of treatment with SKP-SC or media in Experiment 1, there were no significant differences between groups of rats that received CsA and those that did not on any of the behavioral, electrophysiological, or histological outcomes except the RST axon density analysis. Therefore, we pooled data from the CsA and no CsA subgroups to compare all of the animals that received SKP-SCs to all mediatreated animals in all Experiment 1 analyses except the RST measures, where CsA treatment was included as an independent variable.

Rats treated with SCs show improved functional recovery versus media or fibroblast controls

Given that the RST is highly involved in forelimb movement and overground locomotion in the rat (Muir and Whishaw, 2000), we
Behavior: Cylinder Test

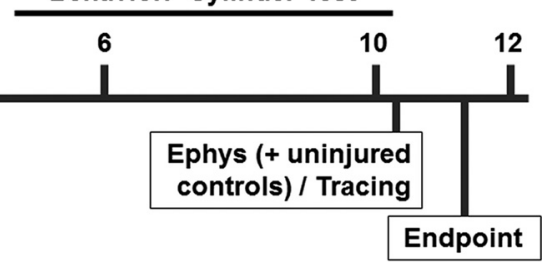

Behavior: Cylinder Test and CatWalk

Figure 1. Experimental design. $\boldsymbol{A}$, Experiment 1 timeline and details. Behavioral testing (cylinder test) was conducted at 6 and weeks after left C4/5 DLF crush injury and immediate treatment with SKP-SCS or media in animals with or without CSA (preinjury and 1 and 8 wpi testing). Uninjured control animals were included in electrophysiological testing during both experiments, but were only traced and perfused alongside treated animals in Experiment 2.

chose to examine the behavioral outcomes of acute cellular therapy after incomplete cervical injury using two separate tests of forelimb function: a relatively simple test of gross forelimb use (cylinder test) and a more detailed analysis of locomotion (CatWalk).

\section{Cylinder test}

We investigated total use of the forelimb affected by injury by analyzing the percent use of "left plus both" forelimbs during vertical exploration (i.e., rearing behavior) in the cylinder test in both experiments (Fig. 2A,B). Consistent with previous studies (Schallert et al., 2000; Bretzner et al., 2008, 2010), we found that uninjured control animals used their left forelimb or both forelimbs on $70-75 \%$ of contacts (dashed lines in Fig. $2 A$ ), as did the animals in Experiment 2 before injury (Fig. 2B, Preinjury). At 6 wpi, the percent use of left plus both decreased to a mean of $\sim 55 \%$ in Experiment 1 regardless of treatment, but at 10 wpi, the SKP-SC group used the left forelimb at significantly greater frequency than the media group $\left(t_{(27)}=-2.27, p=0.031\right.$; Fig. $\left.2 A\right)$, the latter of which exhibited a decline in performance relative to 6 wpi. In Experiment 2, the total use of the left forelimb significantly declined after injury in all groups (see 1 wpi data in Fig. 2B) and remained significantly lower than preinjury values across all subsequent time points tested (RM-ANOVA within-subjects effect, $\left.F_{(4,112)}=10.40, p=0.000\right)$ regardless of treatment. Although both SC groups had higher average total use of the left forelimb than the Fibro group at every time point, there were no significant differences among the treatment groups. Therefore, SKP-SC transplantation resulted in significantly greater use of the forelimb affected by injury compared with media treatment (Experiment 1), but did not improve gross forelimb use relative to $\mathrm{N}-\mathrm{SC}$ or fibroblast transplantation (Experiment 2). It should be noted that the SKP-SC groups in Experiments 1 and 2 demonstrated highly similar mean values at weeks 6 (57 and 55\%, re- 

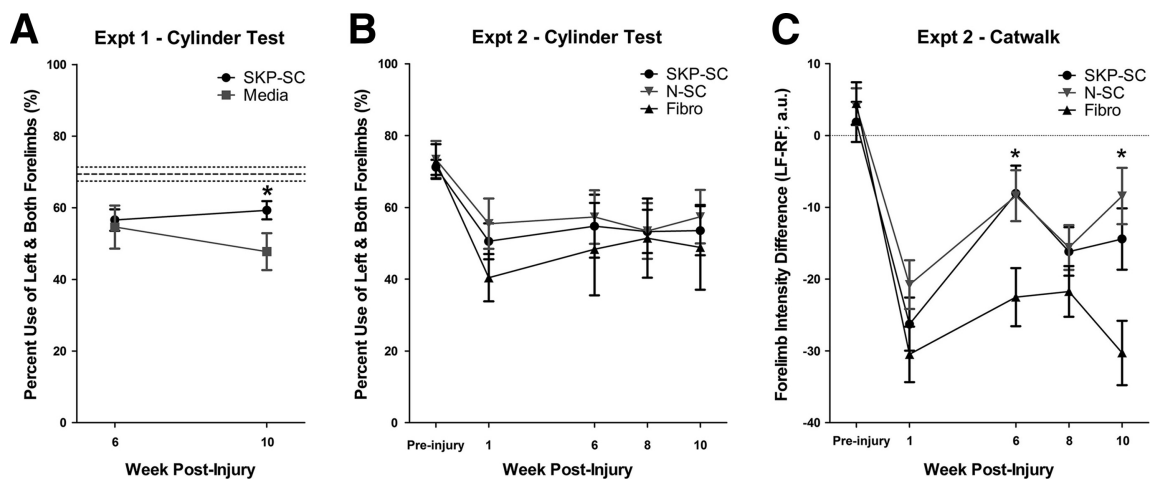

\section{Cell transplantation results in RST motor thresholds and latencies that do not significantly differ from those of uninjured controls}

Given that the RST plays a specific, preferential role in the control of distal forelimb muscles (Küchler et al., 2002), we chose to complement our behavioral assays of forelimb function by assessing RST efficacy using EMG recordings of left forearm extensor muscles during stimulation of the contralateral red nucleus (the origin of the injured RST; see sample trace in Figure $3 A$ ). Motor thresholds and latencies did not differ between uninjured control groups assessed during Experiments 1 and 2 , so we merged all of the data from uninjured animals into one group for comparison with the treatment groups in Experiment 1 and 2 and displayed the results from both experiments on a single graph for motor threshold (Fig. 3B) and latency (Fig. $3 C$ ) measures. The uninjured group was the only group to be compared across experiments here because we other-

spectively) and 10 (59 and 54\%, respectively) because this indicates that transplanted SKP-SCs showed similar behavioral effects across the two studies. That being said, the total use of the left forelimb in Experiment 2 varied more within each group, which may reflect greater variability in injury severity or differences in behavioral scoring across the experiments.

\section{CatWalk analysis}

Analyses of various forelimb gait parameters, including base of support, stride length, paw angle, regularity index, print area, and intensity, from the CatWalk data collected in Experiment 2 (a total of 16 RM-ANOVAs) revealed no significant group differences. However, treatment-related effects were found on forelimb intensity difference scores (left forepaw intensity minus right forepaw intensity). Intensity is a measure of the pressure applied by each paw (Vrinten and Hamers, 2003), so we interpreted this as a difference in weight support provided by the forelimb affected by injury (left) and the forelimb not affected by injury (right). Forelimb intensity difference displayed a significant negative shift after injury, indicating that less weight was distributed to the left forelimb relative to the right. Although this difference in relative weight support was maintained for the duration of the experiment in all animals regardless of treatment (RM-ANOVA within-subjects effect, $\left.F_{(4,124)}=37.14, p=0.000\right)$, there were significant differences among the treatment groups over time as well (RM-ANOVA interaction, $\left.F_{(8,124)}=2.30, p=0.025\right)$. Follow-up ANOVAs revealed significant group differences at 6 and $10 \mathrm{wpi}\left(F_{(2,31)}=\right.$ $4.378, p=0.021$ and $F_{(2,31)}=6.936, p=0.003$, respectively), and Bonferroni post hoc tests revealed that both SKP-SC and $\mathrm{N}-\mathrm{SC}$ groups had significantly smaller forelimb intensity difference scores than the Fibro group at 6 wpi $(p=0.044$ and $p=0.041$, respectively) and $10 \mathrm{wpi}(p=0.047$ and $p=0.003$, respectively); whereas the SKP-SC and N-SC groups did not show significant differences at any time point. Therefore, rats treated with either type of SC distribute their weight more evenly on the forelimbs after injury than rats treated with fibroblasts. wise conducted independent analyses comparing treatment groups within (not between) each experiment.

The efficacy of the RST was generally diminished after DLF crush injury compared with uninjured controls; at $10 \mathrm{wpi}$, the injured animals required stronger stimulation of the red nucleus to evoke motor and EMG responses in the distal forelimb muscles and these signals often took longer to reach target muscles. In addition, rats that required stronger stimulation to trigger an EMG response tended to have longer latencies (Fig. 3D), as indicated by a significant positive correlation between these two measures (Spearman's $\rho=0.333, p=0.012$ ) across all groups (Fig. $3 D$ ). Comparing the treated rats from Experiment 1 with the uninjured controls, we found significant differences among the groups in terms of the threshold of stimulation required to elicit EMGs in the distal musculature $\left(\chi_{(2)}^{2}=10.35, p=0.005\right)$ and the latencies of these responses $\left(\chi_{(2)}^{2}=7.98, p=0.018\right)$. Follow-up tests (adjusted $\alpha=0.0167)$ revealed significantly higher forelimb motor thresholds $\left(U_{(22)}=11.00, p=0.003\right)$ and significantly longer latencies $\left(U_{(19)}=12.00, p=0.009\right)$ in the media group compared with uninjured controls, whereas the SKP-SC-treated animals did not differ significantly from either of these groups (Fig. 3B,C, Experiment 1). In contrast, motor thresholds and latencies did not differ significantly among any of the groups (including uninjured controls) in Experiment 2 (Fig. $3 B, C$, Experiment 2).

\section{Cavitation persists after acute transplantation of SCs, but not fibroblasts}

To characterize the neuroanatomical effects of acute transplantation after unilateral DLF crush injury, we examined lesion and cavity volume as indicators of pathology at 11 wpi. In mediatreated animals, the lesion was characterized by substantial cavitation starting in the GM at the level of the DLF and extending to the edge of the spinal cord through the area normally occupied by the RST (Fig. 4A, Media). A small rim of spared tissue often separated the cavity from the outside of the spinal cord and tissue bridges were occasionally found spanning the ventral portion of the cavity. SKP-SC-transplanted animals showed a similar injury 


\section{A Distal Forelimb EMG}

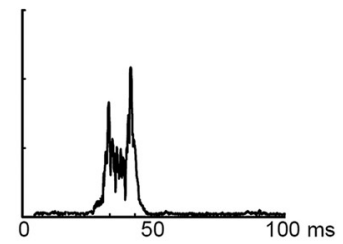

\section{B Distal Forelimb Motor Threshold}

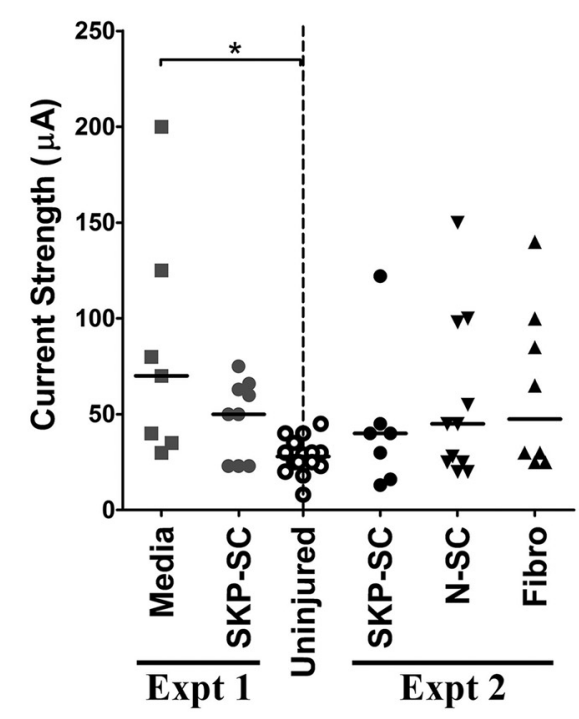

\section{Distal Forelimb EMG Latency}

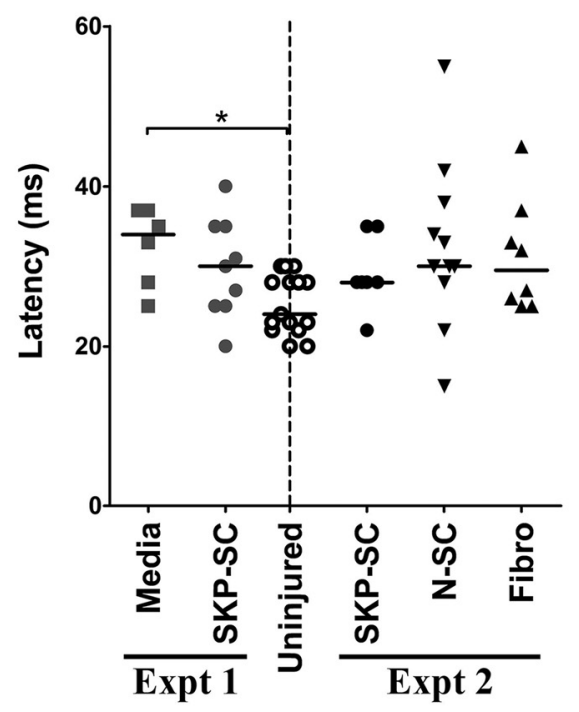

D

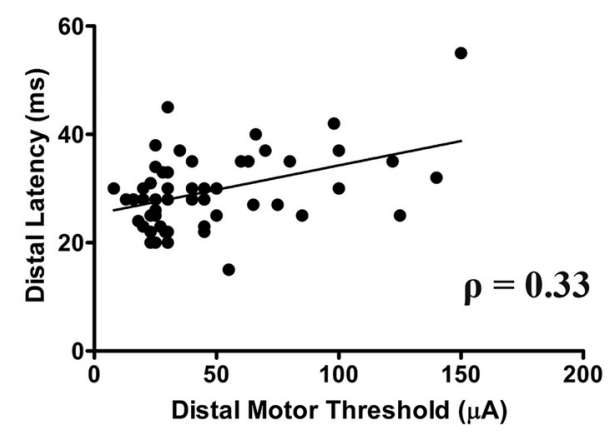

morphology and did not differ significantly from media-treated animals with respect to lesion or cavity volume (Fig. 4A-C, Experiment 1). Midlesion tissue bridges were very common in the SKP-SC groups in both experiments (Fig. $4 A, F^{\prime}, L, L^{\prime \prime}$ ) and similar structures were often observed in the N-SC group in Experiment 2 (Fig. $4 A, F^{\prime \prime}, M, M^{\prime \prime}$ ).

With the exception of cavity volume in the Fibro group, lesion and cavity volumes were generally similar between the two experiments, which suggests approximately equivalent injury severity in both experiments, although severity may have been somewhat more variable in Experiment 2 (cf. Experiments 1 and 2 in Fig. $4 B, C)$. Lesion volume did not differ significantly among the groups in Experiment 2 (Fig. $4 B$ ), but cavity volume did $\left(\chi_{(2)}^{2}=\right.$ $19.44, p=0.000$ ) because the Fibro group showed significantly less cavitation than the SKP-SC or N-SC groups $\left(U_{(17)}=0.00\right.$, $p=0.000$ and $U_{(19)}=0.00, p=0.000$, respectively; adjusted $\alpha=$ 0.0167; see Figure 4C, Experiment 2). Similar results were found when only animals that showed survival of transplant-derived cells were assessed because the percent of lesion occupied by cavity differed significantly among the groups in Experiment 2 $\left(\chi_{(2)}^{2}=20.51, p=0.000\right)$, with the Fibro group showing significantly less cavitation than either the SKP-SC or N-SC groups $\left(U_{(17)}=0.00, p=0.000\right.$ and $U_{(19)}=0.00, p=0.000$, respectively; adjusted $\alpha=0.0167$; Fig. $4 E$, Experiment 2). Indeed, most of the animals (7/9) in the Fibro group showed no cavitation whatsoever because their lesion sites were packed with cells (indicated by nuclear staining in Fig. 4H), extracellular matrix (ECM) molecules such as fibronectin (Fig. 4I), collagen type I (Fig. 4J), laminin (Fig. 4K), and neurocan (Fig. 5B, Fibro), as well as endogenous SCs (labeled with $\mathrm{P}_{0}$ in Fig. $4 G$ ). The lack of cavitation and the presence of cells and ECM in the lesion site occurred in all fibroblast-transplanted animals regardless of graft survival and presumably resulted from the invasion of the lesion site by fibroblasts and other cells that normally reside in the meninges (see also Vroemen et al., 2007). Notably, the general shape of fibroblast-treated lesions was wider and rounder than that observed in other treatment groups (Fig. 4) and a large outgrowth of connective tissue protruded from the injury site and tethered the spinal cord to the surrounding vertebrae in every fibroblasttransplanted animal (data not shown).

\section{Neonatal SCs survive long term after acute transplantation into the crushed DLF}

Most of the animals (15/16 in Experiment 1 and 10/10 in Experiment 2) that received SKP-SC transplants showed some degree of graft survival (Fig. $4 D$ ) at $11 \mathrm{wpi}$, but graft volume was generally low and varied considerably from animal to animal in both

Figure 3. RST efficacy remains at levels no different from uninjured controls after acute cell transplantation into the crushed cervical spinal cord. $A$, EMG response recorded in the left distal forelimb muscles and evoked by stimulation of the contralateral red nucleus. The stimulus artifact has been removed for clarity. $\boldsymbol{B}$, Motor threshold (i.e., minimum current strength in $\mu \mathrm{A}$ ) required to evoke EMG responses in distal forelimb muscles. Only the media-treated group (Experiment 1) differed significantly from uninjured controls at $10 \mathrm{wpi}$, and there were no significant differences among the treated groups in Experiments 1 and 2. C, Latency (in milliseconds) of EMG responses evoked at motor threshold stimulation levels. Only the mediatreated group had significantly longer EMG latencies than the uninjured control group and there were no significant differences among the treated groups in Experiment 1 or 2. D, Scatterplot depicting the relationship between motor threshold and EMG latency for all animals in both experiments. For $\boldsymbol{B}$ and $\boldsymbol{C}$, all results are depicted as individual data points with group medians indicated by solid black lines ${ }^{*} p<0.0167$, Bonferroni-adjusted significance level for Experiment 1 pairwise tests); for $\boldsymbol{D}$, solid black line indicates line of best fit. 
A

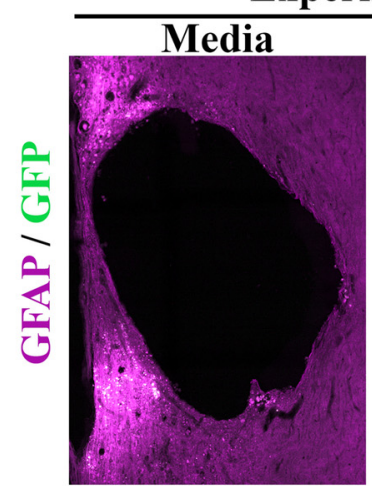

B

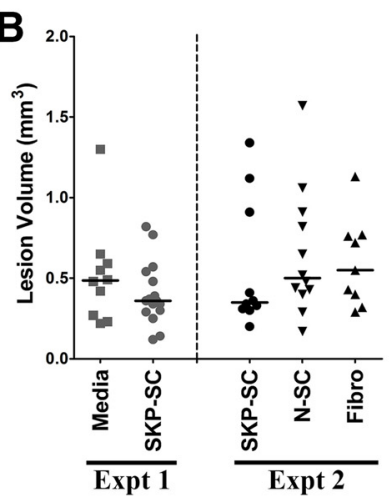

Experiment 1
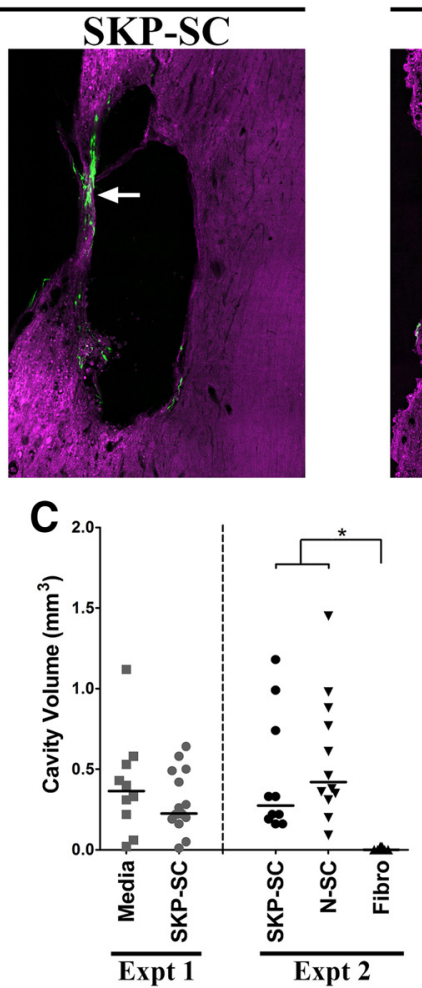

Experiment 2
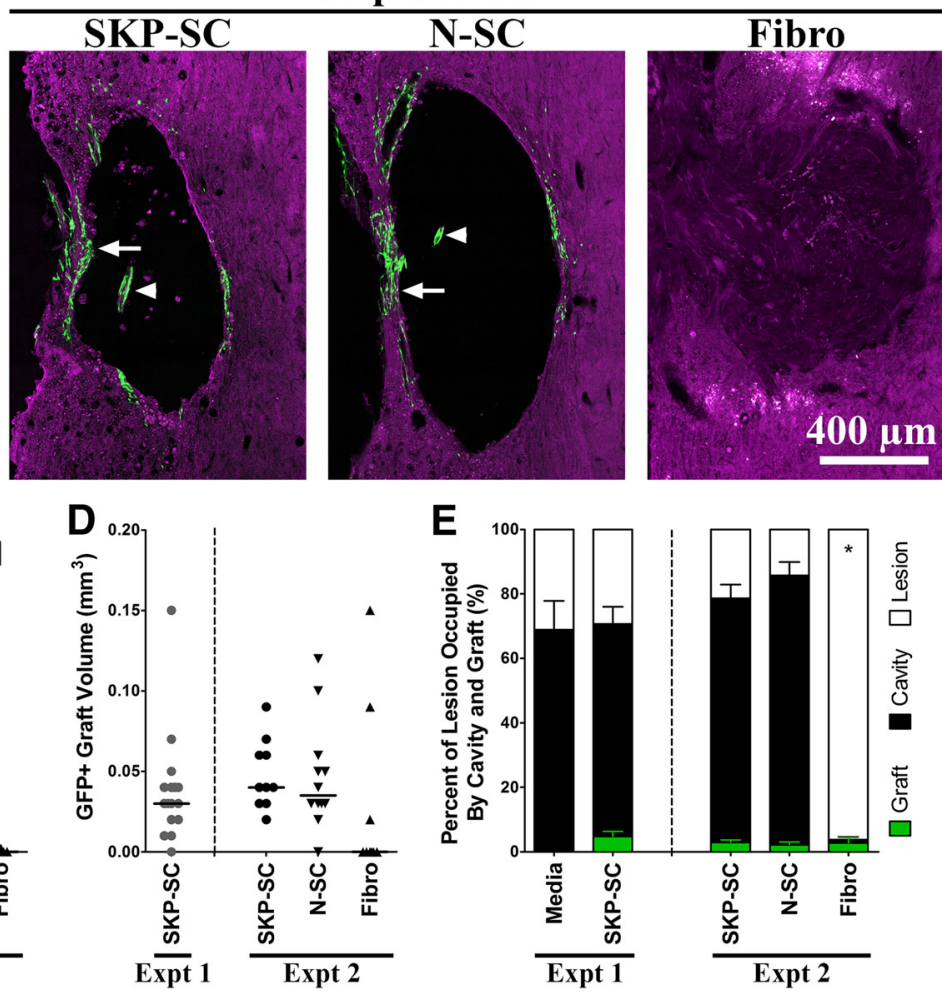
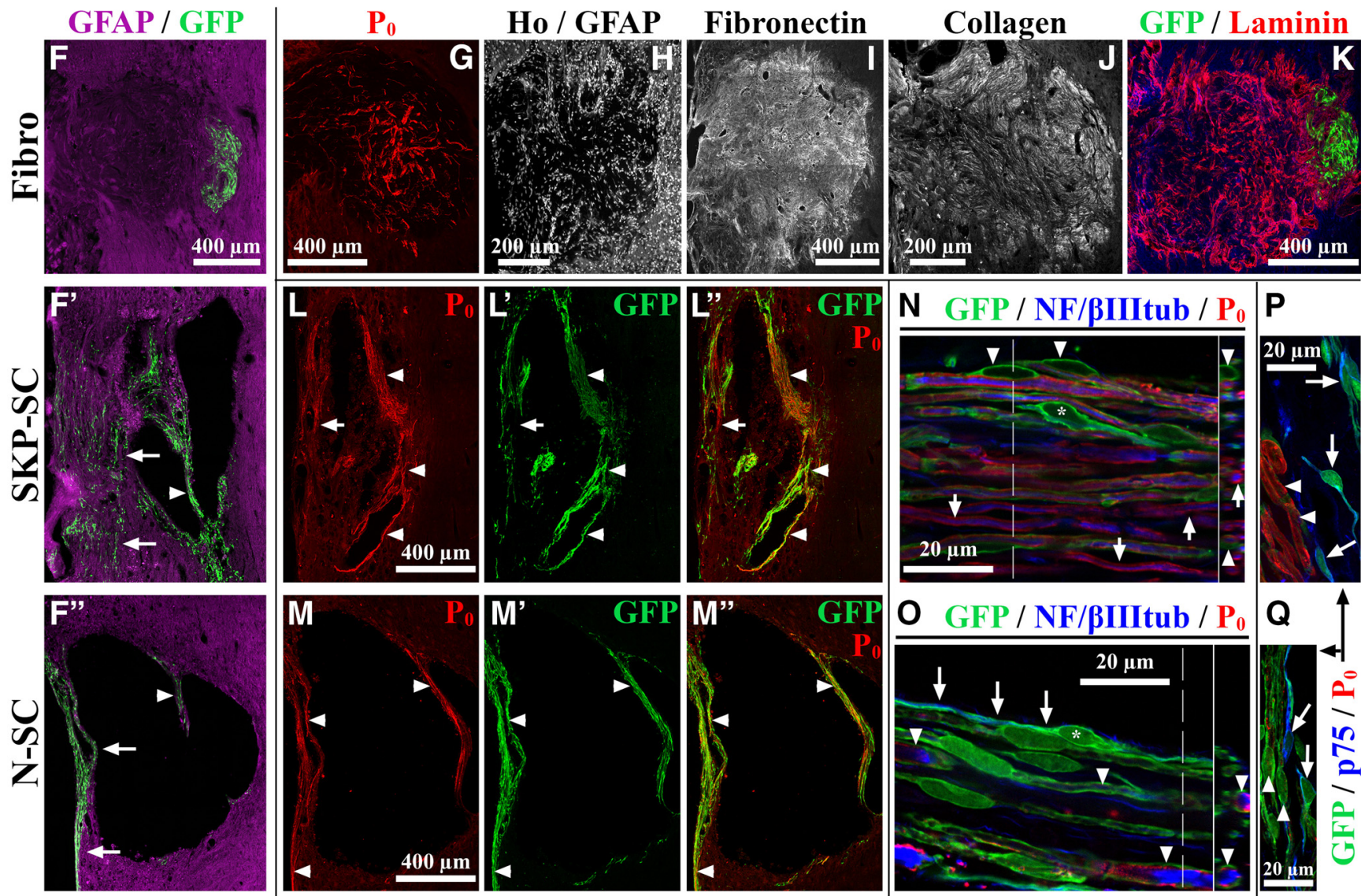

Figure 4. Cellular responses and lesion properties 11 weeks after injury and transplantation. $A$, Representative images (based on median graft/lesion data) of the lesion epicenter in GFAPimmunolabeled (magenta) and GFP-immunolabeled (green) tissue; arrows and arrowheads denote GFP ${ }^{+}$SCs in the spared rim and fragments of midlesion bridges, respectively. $\boldsymbol{B}-\boldsymbol{D}$, Lesion ( $\boldsymbol{B}$ ), cavity ( $\left(\boldsymbol{C}\right.$, and graft (D) volume $\left(\mathrm{mm}^{3}\right)$ data (group medians depicted as solid black lines). $\boldsymbol{E}$, Percentage of lesion (white) occupied by cavity (black) and graft (green) (depicted as means \pm SEM) for tissue containing GFP ${ }^{+}$cells at endpoint. The Fibro group often lacked any GFP ${ }^{+}$cell survival (D) and showed significantly reduced cavitation $(\boldsymbol{C})$ compared with either (Figure legand continues) 
the SKP-SC and N-SC groups (cf. Fig. $4 A, F^{\prime}, F^{\prime \prime}$ ). Curiously, graft volume was not significantly different in SKP-SC-transplanted animals that received CsA versus those that did not (Experiment 1; data not shown) and the failure of CsA to improve cell survival was confirmed in Experiment 2 because SKP-SC graft volume did not differ significantly between the two experiments (Fig. 4D). Graft volume also did not differ significantly between the SKP-SC and N-SC groups in Experiment 2 (Fig. 4D) and the largely overlapping range of volumes in these two groups suggests that transplant-derived SCs from both sources show approximately similar levels of survival after acute transplantation after cervical SCI.

Many fibroblast-transplanted animals (6/9) had no GFP ${ }^{+}$ cells within the spinal cord at $11 \mathrm{wpi}$, but when transplanted fibroblasts did survive, the vast majority were found in the lesion site rather than the surrounding parenchyma, where they occupied a percentage of the lesion volume that did not differ significantly from that occupied by transplanted SCs (Fig. 4E, Experiment 2). $\mathrm{GFP}^{+}$fibroblasts were often found in the meninges outside of the spinal cord as well, suggesting that transplantderived fibroblasts may have survived and migrated out of the spinal cord in some cases (data not shown). In contrast, regardless of source, transplanted SCs shared a common graft morphology wherein cells were primarily found surrounding the edges of the cavity, particularly within the spared rim of tissue at the margin of the spinal cord (arrows in Fig. 4A, SKP-SC and N-SC images, and $F^{\prime}, F^{\prime \prime}$; also visible in Fig. $\left.4 L, L^{\prime \prime}, M, M^{\prime \prime}\right)$ or within tissue bridges spanning the lesion site (arrowheads in Fig. $4 A$, SKP-SC and N-SC images, and $F^{\prime}, F^{\prime \prime}$; also visible in Fig. $\left.4 L, L^{\prime \prime}, M, M^{\prime \prime}\right)$.

\section{Transplanted and endogenous SCs myelinate host axons in the injured cervical spinal cord}

Transplant-derived $\left(\mathrm{GFP}^{+}\right)$SKP-SCs and N-SCs were commonly associated with $\mathrm{P}_{0}{ }^{+}$myelin ensheathed NF/ $\beta$ IIItub ${ }^{+}$axons (arrowheads in Fig. $4 N, P, O, Q$, respectively) in the injured cervical spinal cord. The majority of this myelination occurred in a rostral-caudal orientation throughout the spared rim and midlesion tissue bridges. The SKP-SC- and N-SC-transplanted groups did not differ significantly with respect to the percent of $\mathrm{GFP}^{+}$area containing $\mathrm{P}_{0}{ }^{+}$myelin structures $(31 \pm 7 \%$ and $26 \pm$ $2 \%$, respectively). Intermingled with the transplant-derived myelinating SCs, we found endogenous SCs (arrows in Fig. $4 N$ ) as well as nonmyelinating $\left(\mathrm{P}_{0}{ }^{-} / \mathrm{p} 75^{+}\right)$transplant-derived $\left(\mathrm{GFP}^{+}\right)$

\footnotetext{
$\leftarrow$

(Figure legand continues.) SC group (Experiment 2), but when fibroblasts survived transplantation, they occupied an average percentage of lesion volume similar to that occupied by transplanted SCS $(\boldsymbol{E}) . \boldsymbol{F}, \boldsymbol{F}^{\prime \prime}$, Sample images of GFAP-immunolabeled (magenta) and GFP-immunolabeled (green) tissue depicting higher than median graft survival in Experiment 2 groups; SCs preferentially occupied tissue in the spared rim (arrows) and midlesion bridges (arrowheads). $\mathbf{G}-\boldsymbol{K}$, Sample images of Fibro-transplanted tissue. Regardless of graft survival, fibroblast transplantation resulted in filling of the lesion cavity by endogenous cells (indicated by Hoechst nuclear staining $[\boldsymbol{H}]$ and including $\mathrm{P}_{0}{ }^{+} \mathrm{SCS}[\boldsymbol{G}]$ ) and ECM molecules (including fibronectin [I], collagen type-I [J], and laminin [red; $\boldsymbol{K}]$ ). $\boldsymbol{L}-\mathbf{Q}$, Fluorescent $\left(\boldsymbol{L}, \boldsymbol{M}^{\prime \prime}\right)$ and/or confocal $(\boldsymbol{N}-\mathbf{Q})$ micrographs of GFP-immunolabeled (green) and $\mathbf{P}_{0}$-immunolabeled (red) tissue from SKP-SC-treated $\left(\boldsymbol{L}, \mathbf{L}^{\prime \prime}, \boldsymbol{N}, \boldsymbol{P}\right)$ and N-SC-treated $\left(\boldsymbol{M}, \boldsymbol{M}^{\prime \prime}, \mathbf{O}, \mathbf{Q}\right)$ animals with or without NF/ $\beta$ Illtub immunolabeling (blue; axons; $\boldsymbol{N}, \mathbf{0}$ ) or p75 immunolabeling (blue; $\boldsymbol{P}, \mathbf{Q}$ ). Endogenous $\left(\mathrm{P}_{0}{ }^{+} / \mathrm{GFP}{ }^{-}\right) \mathrm{SC}$ s were occasionally found in both groups (e.g., arrows in $\left.L, \mathbf{L}^{\prime \prime}, \boldsymbol{N}\right)$, but the majority of $\mathrm{P}_{0}{ }^{+} \mathrm{SC}$ myelin was found in areas occupied by transplant-derived cells (arrowheads in $\mathbf{L}-\mathbf{Q}$ ). Transplanted SKP-SCS and N-SCS both generated nonmyelinating $\left(\mathrm{p} 75^{+} / \mathrm{P}_{0}^{-} ;\right.$asterisks in $\boldsymbol{N}, \mathbf{0}$; arrows in $\left.\mathbf{O}-\mathbf{Q}\right)$ and myelinating $\left(\mathrm{P}_{0}{ }^{+} / \mathrm{p} 75^{-}\right.$; arrowheads in $\boldsymbol{N}-\boldsymbol{Q}$ ) SCs in vivo. For $\boldsymbol{B}-\boldsymbol{E},{ }^{*} p<0.0167$ (Bonferroni-adjusted significance level for Experiment 2 pairwise tests) for cavitation measures only.
}

SCs (arrows in Fig. 4O-Q; asterisks in Fig. 4N,O) in both SKPSC- and N-SC-treated spinal cords.

The majority of $\mathrm{P}_{0}{ }^{+}$structures found after acute transplantation of SKP-SCs or N-SCs in the crushed cervical spinal cord were in regions occupied by transplant-derived $\mathrm{GFP}^{+}$cells (Fig. $4 L, L^{\prime \prime}$ for SKP-SC and Fig. $4 M, M^{\prime \prime}$ for N-SC). As an estimate of the contribution of endogenous SCs, we compared the percentage of $\mathrm{P}_{0}{ }^{+}$area that was also $\mathrm{GFP}^{-}$in SC-transplanted animals in Experiment 2 and found that only $\sim 35-40 \%$ of the $\mathrm{P}_{0}{ }^{+}$area was GFP $^{-}$(SKP-SCs: $40 \pm 6 \%$; N-SCs: $35 \pm 3 \%$ ). These data indicate that the majority of SC myelin $(\sim 60-65 \%)$ came from transplant-derived cells in both the SKP-SC and N-SC groups.

We also found a substantial endogenous $\left(\mathrm{P}_{0}{ }^{+} / \mathrm{GFP}^{-}\right)$population of myelinating SCs in fibroblast-transplanted rats, but these cells were almost exclusively restricted to the lesion site and showed a random organization (Fig. $4 G$ ) rather than the rostralcaudal orientation associated with $\mathrm{P}_{0}{ }^{+} \mathrm{SC}$ myelin in the spared rim and lesion bridges of SKP-SC- and N-SC-transplanted rats (Fig. $4 L, M$, respectively). In contrast, we found little $\mathrm{P}_{0}{ }^{+}$myelin in media-treated animals (data not shown), which is consistent with previous evidence that the endogenous SC response is more robust in the presence of transplanted SCs (Hill et al., 2006; Biernaskie et al., 2007).

\section{Cell transplantation does not increase GFAP or neurocan expression}

We assessed the degree of reactive astrogliosis in response to treatment after incomplete cervical SCI using immunoreactivity as a measure of the expression of GFAP (GFAP-IR) and the CSPG neurocan (neurocan-IR) in tissue immediately rostral or caudal to the lesion site at 11 wpi. Despite using the same reagents and similar histological methods in both experiments, we noted higher GFAP-IR values for animals in Experiment 1 versus Experiment 2 (Fig. $5 C, D$ ). As one would expect after SCI, GFAP-IR and neurocan-IR measures both showed positive normalized values $>1$ (Fig. $5 C-F$ ) in all cases regardless of treatment.

In Experiment 1, we found no significant difference between media-treated and SKP-SC-treated rats in terms of GFAP-IR (Fig. 5A, C,D, Experiment 1) or neurocan-IR (Fig. $5 B, E, F$, Experiment 1) measured rostral or caudal to the injury site. Comparing the different types of cell transplants in Experiment 2 revealed similar findings because there were no significant differences among the treated groups in terms of rostral or caudal normalized GFAP-IR (Fig. $5 A, C, D$, Experiment 2) or neurocan-IR (Fig. $5 B, E, F$, Experiment 2). In addition, we measured GFAP-IR only in regions immediately adjacent to transplanted SKP-SCs or N-SCs (Experiment 2 only) and, again, we found no significant difference between these groups (data not shown). Therefore, contrary to previous reports (Pearse et al., 2004), we found no evidence that transplanted SCs (from either source) enhanced reactive astrogliosis relative to media and/or fibroblast treatment in a model of acute transplantation into partial cervical SCI.

\section{Transplanted SKP-SCs and N-SCs do not differ in terms of migration or integration into host parenchyma in the injured cervical spinal cord}

In Experiment 2, we compared the migration and integration of neonatal rat SKP-SCs and N-SCs after acute transplantation into the crushed cervical DLF. By 11 wpi, nearly all of the surviving SCs from both sources had migrated out of the lesion cavity and into spared, if not completely intact, host parenchyma (Fig. $5 G, H)$. The majority of these cells were found in the spared rim 


\section{Experiment 1}

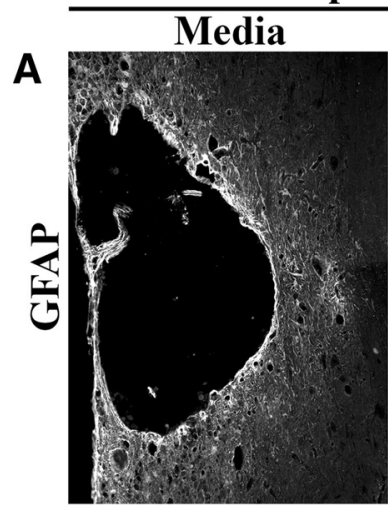

B

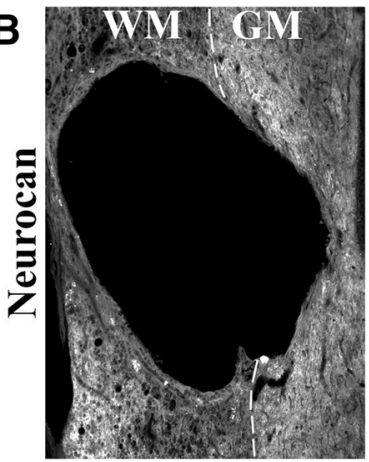

C

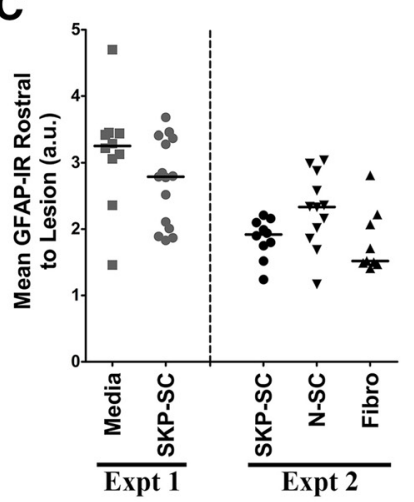

SKP-SC
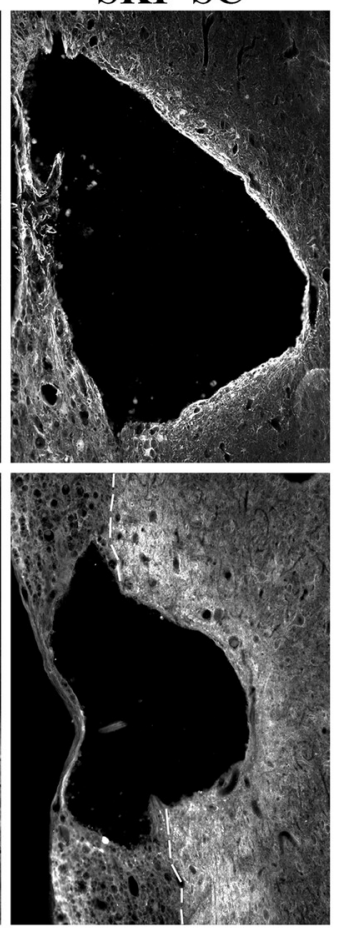

D

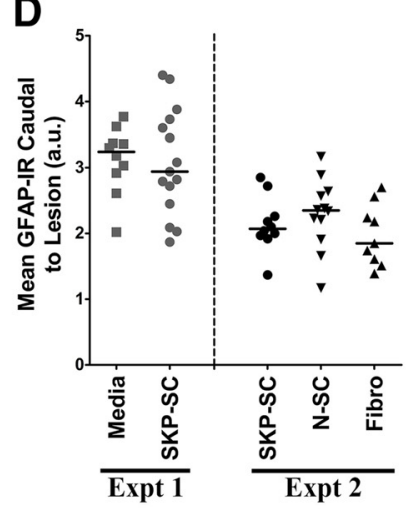

Experiment 2

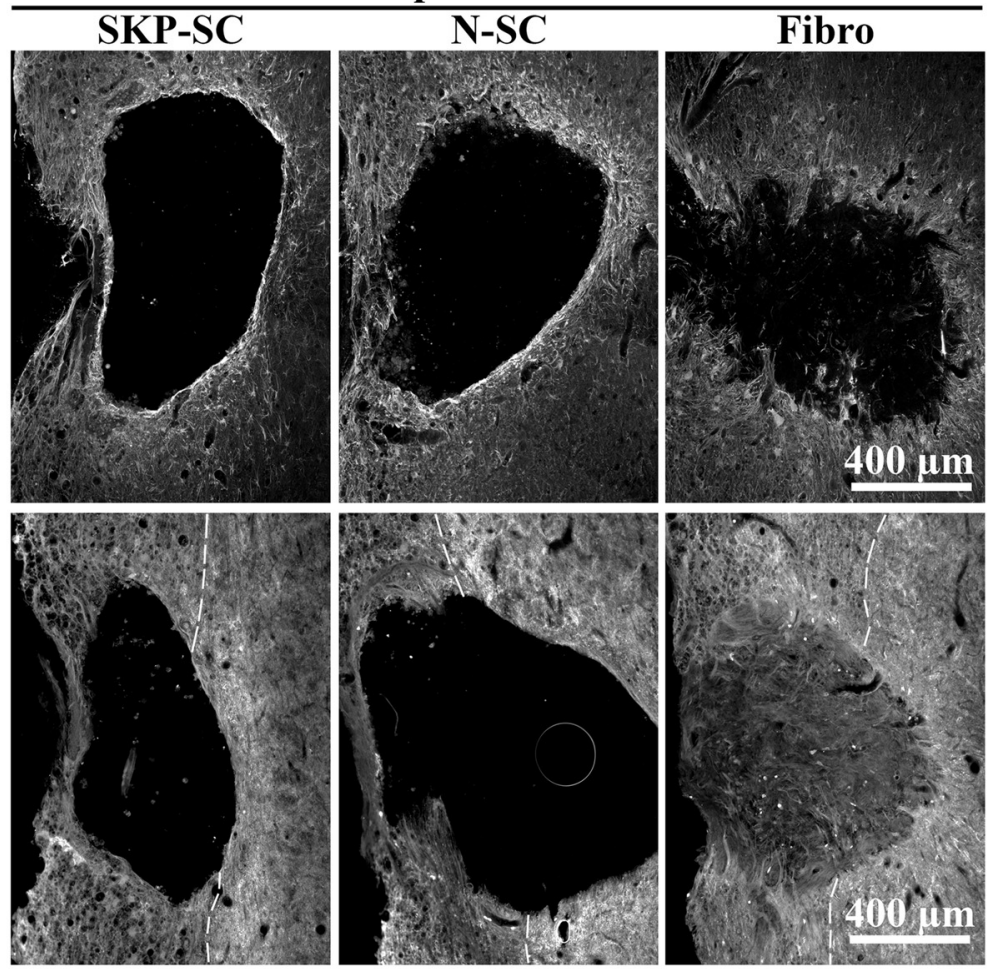

E

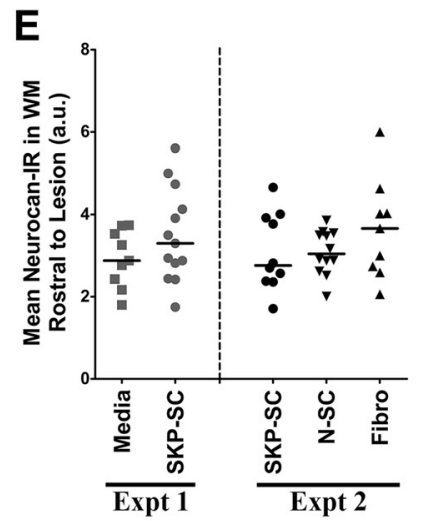

F

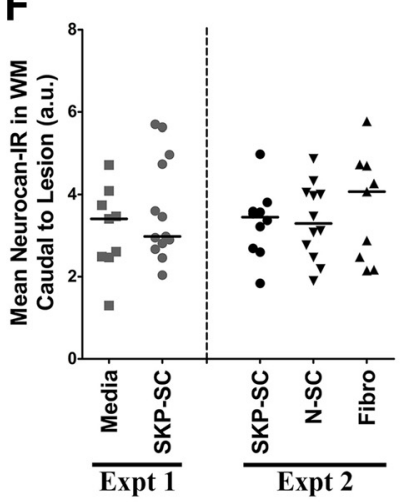

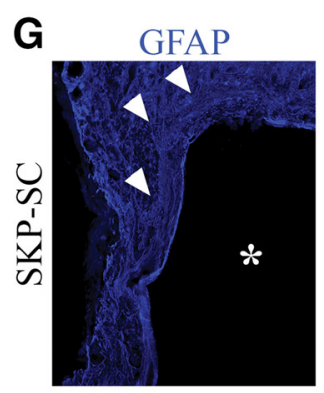

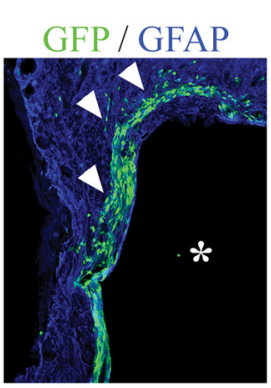

\section{H} GFP / GFAP
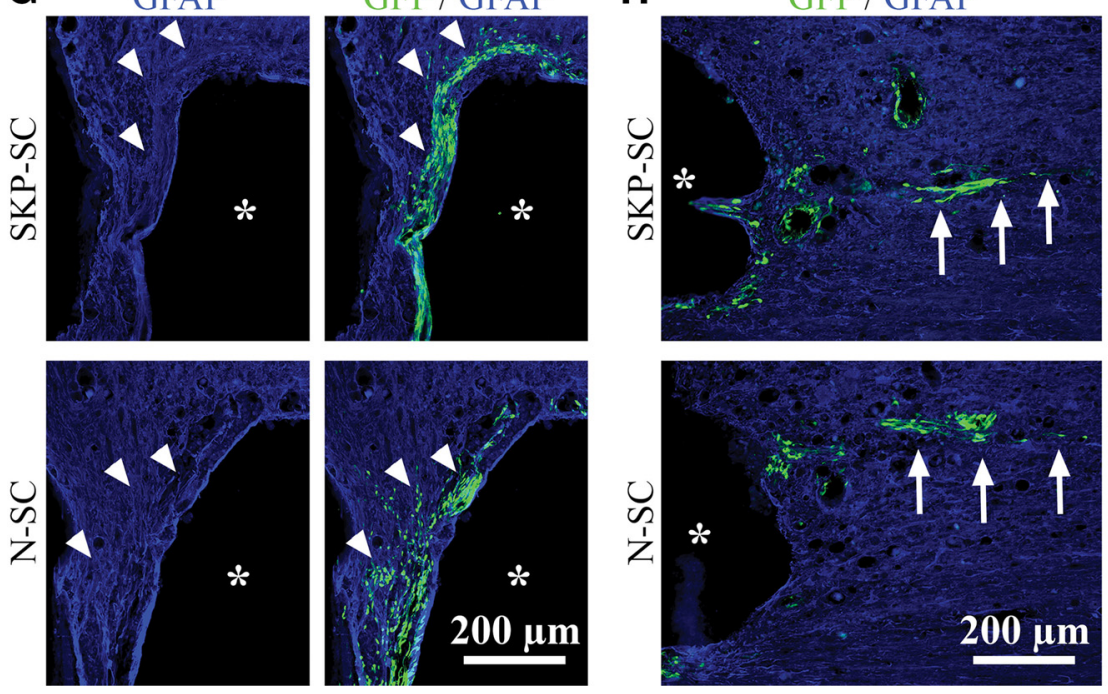

Figure 5. Transplanted SKP-SCs and N-SCs do not enhance reactive astrogliosis or differ in their capacity to migrate or integrate into injured cervical host parenchyma. $\boldsymbol{A}, \boldsymbol{B}$, Representative images of GFAP-immunolabeled ( $\boldsymbol{A})$ or neurocan-immunolabeled $(\boldsymbol{B})$ longitudinal sections at the level of injury at 11 wpi for each group in Experiment 1 (Media and SKP-SC) and (Figure legand continues) 
and midlesion bridges, where $\mathrm{GFAP}^{+}$cells are present, but display an abnormal cytoarchitecture indicative of "lesioned" tissue. However, SCs from either source were often found in relatively intact $\mathrm{GFAP}^{+}$host parenchyma as well (arrowheads in Fig. 5G) and transplant-derived cells that had migrated further out from the lesion site into relatively normal host tissue were observed occasionally (arrows in Fig. 5H). Quantification of the area outside of the lesion site occupied by SCs demonstrated no significant difference between SKP-SCs and N-SCs in terms of their integration with host astrocytes (data not shown). Small numbers of SCs were commonly found lining the outside of the spinal cord near the injury site and a few were also observed within the central canal further from the lesion site in some animals (data not shown). Ignoring these cells, we found no significant difference between SKP-SCs and N-SCs when we assessed the maximum distance of SC migration through astrocyte-rich host parenchyma (data not shown).

\section{SKP-SC-transplanted rats show increased density of RST fibers in the injured cervical spinal cord relative to other treatment groups}

Assessments of BDA-labeled RST axons revealed no evidence of sparing or regeneration in the WM. To assess RST collaterals in the GM, we analyzed the raw BDA-traced RST axon density in the ipsilateral spared GM at $\mathrm{C} 2$, far rostral ( $\sim 1 \mathrm{~mm}$ rostral), immediately rostral, and immediately caudal to the injury site (depicted in Fig. $6 A$ ) or C4/5 in uninjured controls. BDA-traced RST axons were found descending in the DLF of the WM at C2 in all animals and these axons projected into the GM to some extent at all levels of analysis and in each group (Fig. 6B, images depicting near-median $\mathrm{BDA}^{+}$axon densities). Preliminary analysis of the $\mathrm{BDA}^{+}$axon counts in the $\mathrm{WM}$ at $\mathrm{C} 2$ revealed that the rats traced in Experiment 1 had significantly higher axon counts than those traced in Experiment $2\left(t_{(52)}=-4.24, p=0.000\right.$; data not shown), and that finding was confirmed by comparing the two (SKP-SC) groups that received the same treatment in both experiments $\left(U_{(20)}=16.00, p=0.010\right.$; compare SKP-SC groups in Fig. $6 C, H)$. As a result of this difference in the efficacy of BDA tracing between Experiments 1 and 2, RST axon counts and densities could not be meaningfully compared across the two experiments and the uninjured controls (traced alongside rats in Experiment 2) could not be compared with treated rats in Experiment 1.

There were no significant differences in RST axon counts among the groups in Experiment 1 (media or SKP-SC) regardless of CsA administration (Fig. 6C). However, there were significant differences on the RST axon density measures among the treatment groups at far rostral $\left(\chi_{(3)}^{2}=11.95, p=0.008\right)$ and rostral

\footnotetext{
(Figure legand continues.) Experiment 2 (SKP-SC, N-SC, Fibro). In tissue immunolabeled for neurocan $(\boldsymbol{B})$, dashed lines mark the boundary between GM and WM. $\boldsymbol{C}-\boldsymbol{F}, \operatorname{GFAP}-\mathrm{IR}(\boldsymbol{C}, \boldsymbol{D})$ and neurocan-IR $(\boldsymbol{E}, \boldsymbol{F})$ data for individual animals with the group median depicted as a solid black line. There were no significant differences between the media-treated and SKP-SC-treated groups in Experiment 1 or the SKP-SC, N-SC, and Fibro groups in Experiment 2 on GFAP-IR or neurocan-IR measured rostral $(\boldsymbol{C}, \boldsymbol{E}$, respectively) or caudal $(\boldsymbol{D}, \boldsymbol{F}$, respectively) to the injury site. $\boldsymbol{G}, \boldsymbol{H}$, Sample images of GFP ${ }^{+}$(green) SKP-SCs (top), and N-SCs (bottom) that have migrated out of the lesion cavity and into $\mathrm{GFAP}^{+}$(blue) host parenchyma 11 weeks after transplantation into the injured cervical spinal cord. These cells were largely found immediately adjacent to the lesion cavity (asterisks in $\mathbf{G}, \boldsymbol{H}$ ), around the edge of the injury site, or in the spared rim and midlesion bridges, where the GFAP ${ }^{+}$astrocytes display abnormal cytoarchitecture. However, $\mathrm{SCs}$ from both sources were occasionally observed in $\mathrm{GFAP}^{+}$regions with relatively normal cytoarchitecture ( $\boldsymbol{G}$, arrowheads) and small patches of these cells were also observed further out from the lesion site in relatively intact tissue ( $\boldsymbol{H}$, arrows).
}

$\left(\chi_{(3)}^{2}=14.02, p=0.003\right)$ levels of analysis, where follow-up tests (adjusted $\alpha=0.0083$ ) revealed that the only significant differences were between the media-treated and SKP-SC-treated subgroups that did not receive $\operatorname{CsA}\left(U_{(9)}=0.00, p=0.008\right.$ for both; Fig. $6 E, F)$. Therefore, in the absence of CsA, the transplantation of SKP-SCs was associated with significantly increased RST axon density in the GM up to $1 \mathrm{~mm}$ rostral to injury compared with media treatment, but this effect disappeared when the animals being compared were immunosuppressed with CsA. Closer inspection of the median RST axon densities revealed that CsA had opposite effects on RST densities in the media-treated and SKPSC-treated groups, causing a slight increase in the former and a slight decrease in the latter at every level of analysis (Fig. 6D-G). Therefore, CsA administration eliminated differences between the media-treated and SKP-SC-treated subgroups through the combination of nonsignificant and opposing effects on these groups. There was no significant difference between mediatreated and SKP-SC-treated groups with respect to GM sparing adjacent to the lesion in this experiment regardless of CsA treatment (data not shown), so these effects could not be explained in terms of enhanced neuroprotection of the GM.

Experiment 2 was initiated before the analysis of Experiment 1 data was complete, so we administered CsA to all of the animals in the second experiment in an effort to minimize the rejection of transplanted cells. Compared with uninjured control animals, the injured animals in Experiment 2 had fewer BDA ${ }^{+}$RST axons in the WM $\left(U_{(28)}=17.00, p=0.004\right)$ and lower GM RST axon density at every level below C2 (far rostral: $U_{(33)}=16.00, p=$ 0.002; rostral: $U_{(33)}=20.00, p=0.003$; caudal: $U_{(33)}=1.00, p=$ 0.000 ; adjusted $\alpha=0.01$; data not shown), and this was especially obvious at the caudal level (Fig. 6B, Experiment 2 images). As mentioned in the Materials and Methods section, the number of $\mathrm{BDA}^{+}$RST axons in the WM at C2 differed among the groups (including uninjured controls) in Experiment $2\left(\chi_{(3)}^{2}=7.77, p=\right.$ 0.020; Fig. $6 H$ ). Follow-up testing demonstrated that RST axon counts were significantly lower in the Fibro group than in the uninjured control group and the SKP-SC group $\left(U_{(12)}=0.00\right.$, $p=0.002$ and $U_{(13)}=2.00, p=0.003$, respectively; adjusted $\alpha=$ $0.0083)$. In addition, the RST axon densities in Experiment 2 differed significantly among the groups (including uninjured controls) at all levels of analysis $\left(\mathrm{C} 2: \chi_{(3)}^{2}=15.76, p=0.001\right.$; far rostral: $\chi_{(3)}^{2}=18.11, p=0.000$; rostral: $\chi_{(3)}^{2}=16.83, p=0.001$; caudal: $\chi_{(3)}^{2}=17.01, p=0.001$; Fig. $\left.6 I-L\right)$. Follow-up testing (adjusted $\alpha=0.0083$ ) revealed that the SKP-SC group did not differ significantly from the uninjured group at any level of testing rostral to the injury, whereas the uninjured group had significantly higher RST densities than the Fibro group at $\mathrm{C} 2\left(U_{(13)}=\right.$ $0.000, p=0.001)$ and the N-SC and Fibro groups at far rostral $\left(U_{(14)}=2.00, p=0.002\right.$ and $U_{(13)}=1.00, p=0.002$, respectively) and $\operatorname{rostral}\left(U_{(14)}=4.00, p=0.005\right.$ and $U_{(13)}=2.00, p=0.003$, respectively) levels of measurement (Fig. $6 I-K$ ). In addition, the SKP-SC group also had significantly higher RST densities than the Fibro group at these levels $\left(\mathrm{C} 2: U_{(17)}=11.00, p=0.006\right.$; far rostral: $U_{(17)}=11.00, p=0.006$; rostral: $U_{(17)}=11.00, p=0.006$; Fig. $6 I-K)$. In contrast, all of the injured groups showed a large and significant decline in RST axon density in the GM caudal to injury compared with the analogous level of assessment (caudal to $\mathrm{C} 4 / 5)$ in the uninjured controls (SKP-SC: $U_{(14)}=0.00, p=$ 0.001 ; N-SC: $U_{(14)}=1.00, p=0.002$; Fibro: $U_{(13)}=0.00, p=$ 0.001; Fig. $6 L$ ).

These effects appeared to be functionally relevant because we found a significant positive correlation between the CatWalk intensity differences measured at $10 \mathrm{wpi}$ and both WM axon counts 
A

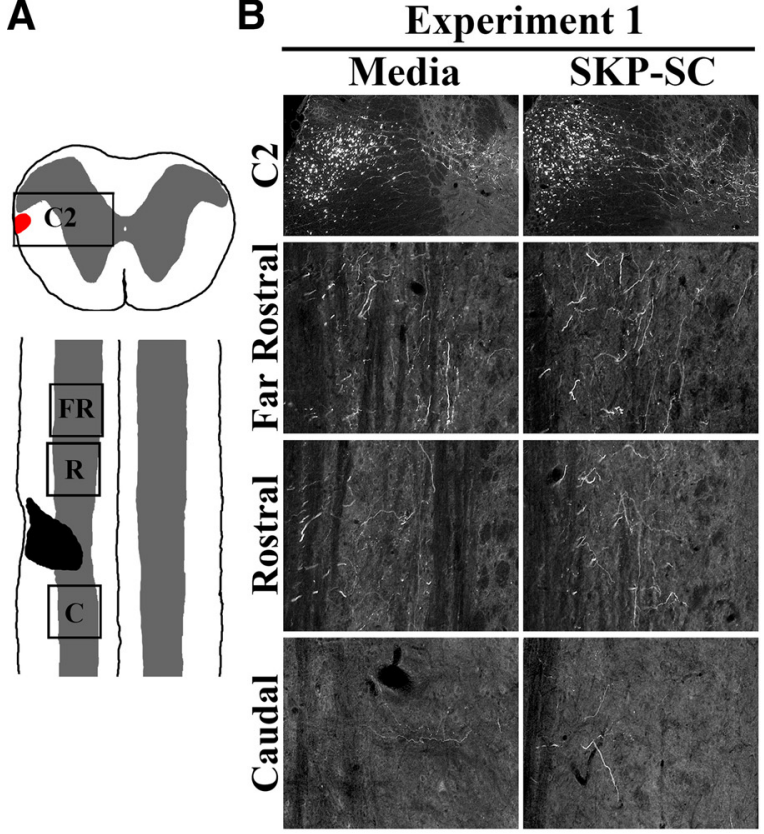

Experiment 2

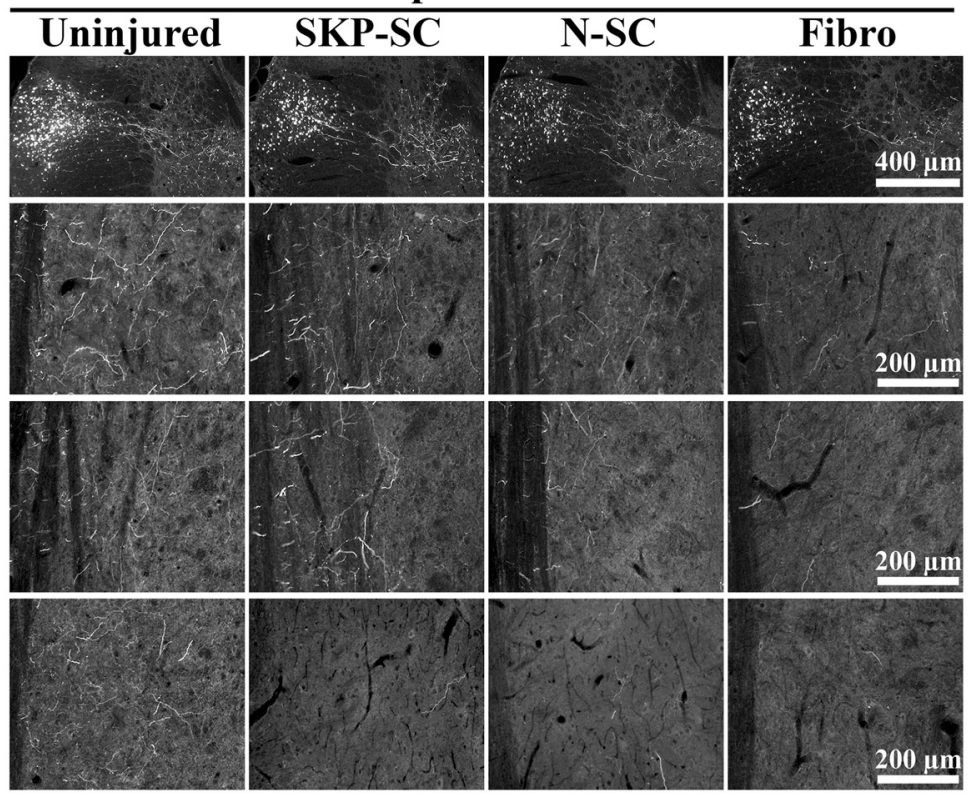

E $\underline{\text { Far Rostral }}$

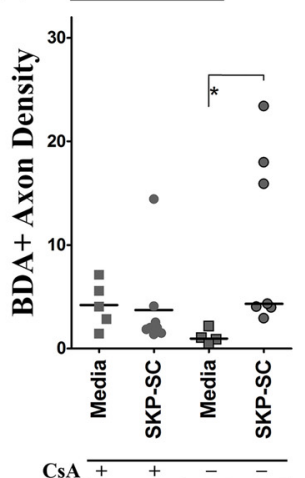

F $\quad \underline{\text { Rostral }}$
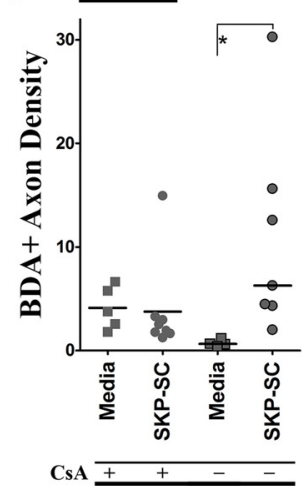

G Caudal

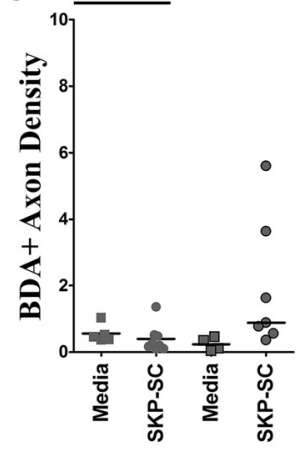

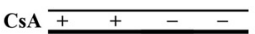

L Caudal

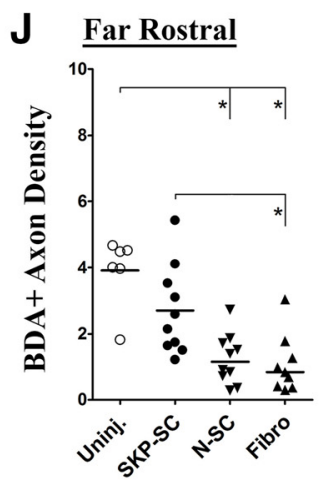

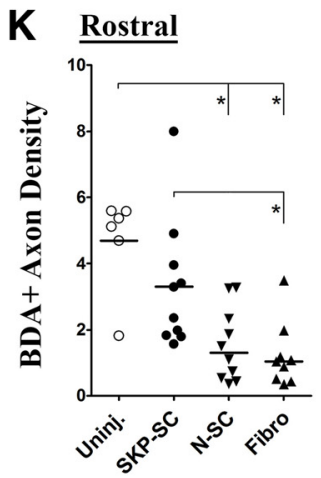

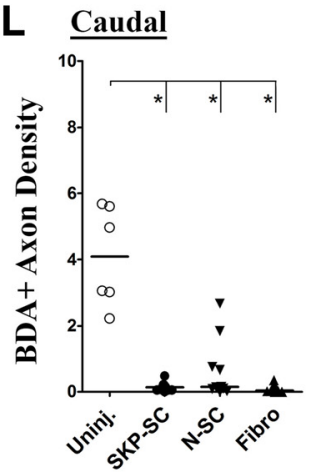

Figure 6. Rats transplanted with SKP-SCS show elevated RST axon counts or densities relative to other treatment groups. A, Diagram depicting coronal section (at (2) and cervical region of the injured rat spinal cord after a left C4/5 DLF crush. The red area in the coronal section represents the RST, which was completely ablated by the injury at C $4 / 5$ in all tissue examined. The injury site (black) encompassed nearly all of the WM (white) and a portion of the GM (gray) at C4/5. BDA-labeled RST axon counts and/or densities were quantified at C2, far rostral (FR), immediately rostral (R), and immediately caudal (C) to the injury site (boxed regions). B, Sample images depicting near-median BDA-labeled RST axon densities in coronal (C2) or longitudinal (far rostral, rostral, caudal) sections at 11 wpi for each group. $\boldsymbol{C}-\boldsymbol{L}$, Individual RST axon count/density data (Experiment 1: $\boldsymbol{C}-\boldsymbol{G}$; Experiment 2: $\boldsymbol{H}-\boldsymbol{L}$; group medians depicted as solid black lines), including BDA ${ }^{+}$axon counts in the WM at $C 2(\boldsymbol{C}, \boldsymbol{H})$ and raw $\mathrm{RST}$ axon density measures in the $G M$ at $(2(\boldsymbol{D}, \boldsymbol{I})$, far rostral $(\boldsymbol{E}, \boldsymbol{J})$, rostral $(\boldsymbol{F}, \boldsymbol{K})$, or caudal $(\boldsymbol{G}, \boldsymbol{L})$ to the lesion site (or $(4 / 5$ in uninjured controls), grouped according to injury/cellular transplantation (and CSA treatment in Experiment 1 only; with CSA [+] and without CSA [-]). In the absence of CsA, the SKP-SC group had significantly greater RST axon density than media controls at far rostral and rostral levels $(\boldsymbol{E}, \boldsymbol{F})$. In the presence of $(S A, R S T$ axon density in the GM did not differ significantly between these two groups at any level (D-G). Compared with the Fibro group in Experiment 2, the uninjured and SKP-SC-treated groups both had significantly higher BDA ${ }^{+}$axon counts $(\boldsymbol{H})$ and significantly higher RST densities at $\mathrm{C2}(\boldsymbol{I})$, far rostral $(\boldsymbol{J})$, and rostral $(\boldsymbol{K})$ to injury. The uninjured group alone also had significantly higher RST axon density than the N-SC group at far rostral ( $)$ and rostral $(K)$ levels. In contrast, the SKP-SC group did not differ from the uninjured group on any analysis rostral to injury, whereas all three transplant groups had significantly lower RST densities caudal to injury than the uninjured $\operatorname{controls}(\boldsymbol{L})$. For $\mathbf{C}-\boldsymbol{L},{ }^{*} p<0.0083(B o n f e r r o n i-$ adjusted significance level for pairwise tests in both experiments). 
A

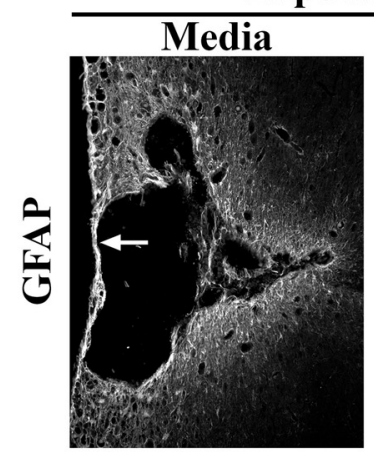

\section{B}

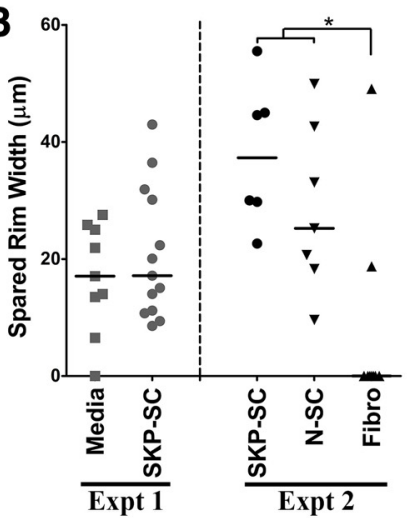

Experiment 1
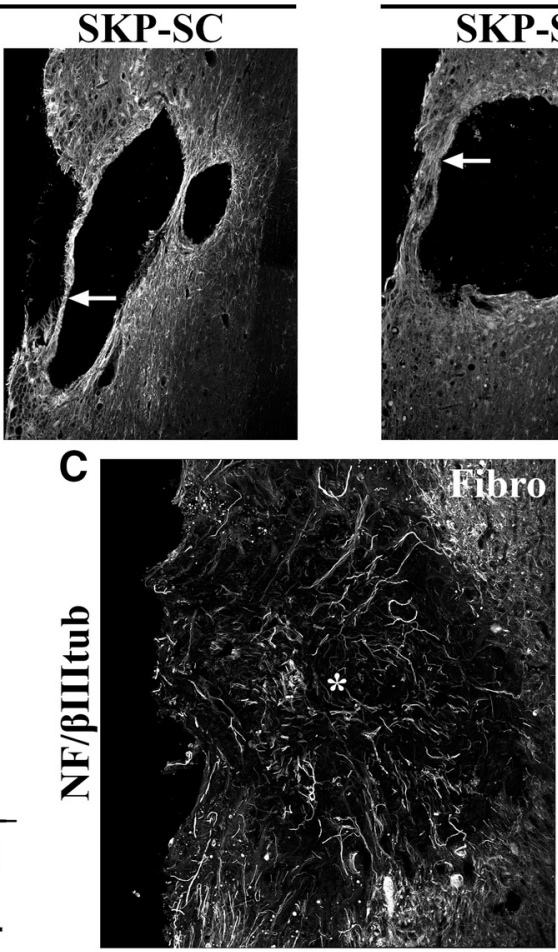

N-SC
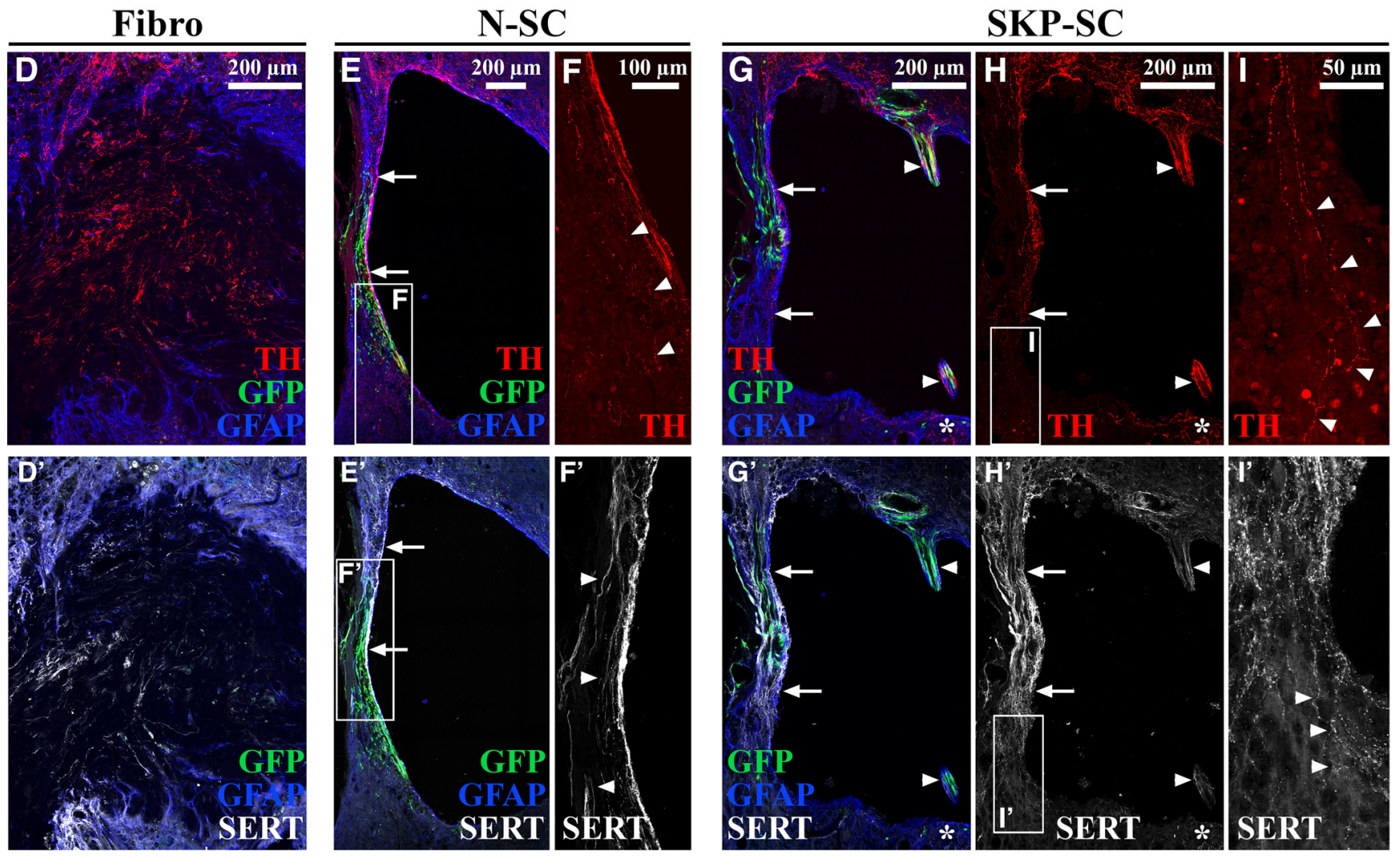

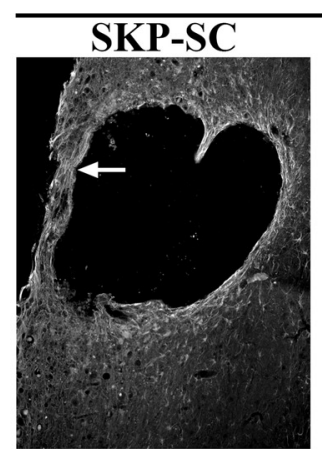

Experiment 2
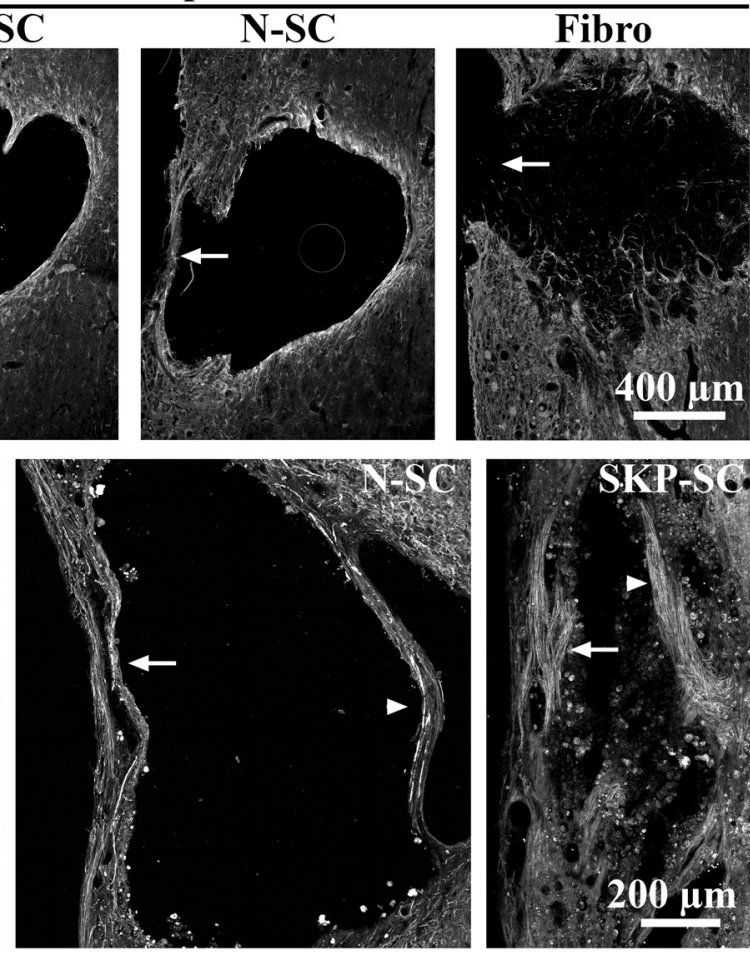

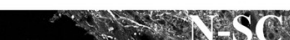

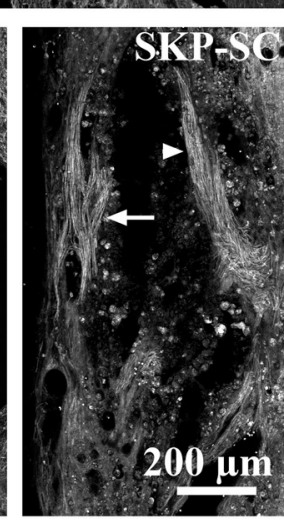

$200 \mathrm{Mm}$

Figure 7. Relative to fibroblasts, transplanted SCs enhance the substrate available for axons to traverse the lesion site from rostral to caudal. $A$, Representative images (based on group median spared rim widths) of GFAP-immunolabeled tissue at the level of injury depicting the relative sizes of the spared tissue rims (arrows) in each treatment group. $\boldsymbol{B}$, Spared rim width ( $\mu \mathrm{m}$ ) depicted as individual data points with group medians indicated by solid black lines $\left({ }^{*} p<0.0167\right.$, Bonferroni-adjusted significance level for Experiment 2 pairwise tests). Both SC groups had significantly thicker spared rims than the Fibro group (Experiment 2) becaue the majority of animals in the latter group had no spared rim at the lesion epicenter $(\boldsymbol{A}, \boldsymbol{B})$. $\boldsymbol{C}$, Sample images of NF/ $\beta \| l$ tub ${ }^{+}$axons in each group in Experiment 2. Axons were oriented rostro-caudal in the spared rim (arrows) and midlesion bridges (arrowheads) of N-SC- and SKP-SC-treated tissue, whereas axons within the lesion site of animals in the Fibro group were oriented toward the middle of the lesion (asterisk in $\boldsymbol{C}$ ) and/or the lateral edge of the spinal cord (i.e., the periphery). $\mathbf{D}-\boldsymbol{I}^{\prime}$, Sample images from animals in each group in Experiment 2 immunolabeled for GFP (green), GFAP (blue), and TH (red; $\boldsymbol{D}-\boldsymbol{H}, \boldsymbol{I}$ ) or SERT (white; $\boldsymbol{D}^{\prime}, \boldsymbol{E}^{\prime}, \boldsymbol{F}^{\prime}, \boldsymbol{G}^{\prime}, \boldsymbol{H}^{\prime}, \boldsymbol{I}^{\prime}$ ). Similar to NF/ $\beta$ Illtub ${ }^{+}$axons $\left(\boldsymbol{C}\right.$ ), TH ${ }^{+}$and SERT ${ }^{+}$axon subpopulations both lacked appropriate rostral-caudal orientation in the Fibro group $\left(\boldsymbol{D}, \boldsymbol{D}^{\prime}\right)$, but crossed the spared tissue rim (arrows in $\boldsymbol{E}, \boldsymbol{E}^{\prime}[\mathrm{N}-\mathrm{SC}]$ and $\mathbf{G}, \boldsymbol{H}, \boldsymbol{G}^{\prime}, \boldsymbol{H}^{\prime}$ (Figure legand continues) 
at C2 (Pearson's $r=0.43, p=0.035$ ) and raw RST axon density in the GM caudal to the lesion (Spearman's $\rho=0.474, p=0.009$ ) among the injured groups in Experiment 2. These correlations indicated that animals with more $\mathrm{BDA}^{+} \mathrm{RST}$ axons at $\mathrm{C} 2$ or higher RST axon density caudal to injury distributed their weight more evenly on the forepaws after injury/treatment. Among the injured/treated animals in Experiment 2, we also noted a significant negative correlation between distal motor threshold and raw RST axon density far rostral and rostral to injury (Spearman's $\rho=-0.571, p=0.0005$ and Spearman's $\rho=-0.558, p=0.007$, respectively), which indicated that animals with higher RST axon density in the GM up to $1 \mathrm{~mm}$ from the lesion required less stimulation to elicit motor responses in the distal forelimb affected by injury. In summary, these results suggest that higher RST axon counts at C2 or higher RST density in the GM at far rostral, rostral, or caudal levels may have all contributed in part to the functional improvements observed for the SKP-SC or N-SC groups relative to the Fibro group.

\section{Rats transplanted with SCs show enhanced preservation of the spared tissue rim and midlesion bridges that provide a substrate for spared and sprouting axons}

In the present study, we measured the width of the spared rim immediately lateral to the injury site in two sections near the lesion epicenter after treatment of a DLF crush at C4/5. With the exception of the Fibro group, we noted that the groups in Experiment 2 tended to have thicker spared rim widths than the groups in Experiment 1 (cf. Experiment 1 and 2 data in Fig. $7 A, B$ ), yet another reason that the data from the two experiments could not be merged. The results of Experiment 1 showed no significant difference between Media-treated and SKP-SC-treated groups overall (Fig. $7 A, B$, Experiment 1). It should be noted that this analysis did not include spared, midlesion tissue bridges because these structures tend to be found in ventral sections, not near the lesion epicenter where this analysis was conducted. In Experiment 2, we found a significant difference among the three treatment groups $\left(\chi_{(2)}^{2}=10.29, p=0.006\right)$ : both SC groups had significantly larger spared rim widths than the Fibro group (SKPSC: $U_{(13)}=5.00, p=0.006$; N-SC: $U_{(14)}=8.00, p=0.009$; Fig. $7 B$, Experiment 2), the latter of which typically lacked any spared tissue rim at the lesion epicenter (Fig. 7A, Experiment 2).

In SKP-SC- and N-SC-treated tissue, NF/ $\beta$ IIItub-positive axons were found traversing both the spared rim (arrows in Fig. $7 C$ ) and tissue bridges spanning the lesion site (arrowheads in Fig. $7 C)$. In sharp contrast, the NF/ $\beta$ IIItub-positive axons in fibroblast-treated animals that lacked a lesion cavity were clearly oriented toward the center of the lesion (asterisk in Fig. 7C) and/or laterally toward the outside of the spinal cord and these axons appeared to have stalled inside the lesion because none were found to traverse the lesion site and exit into spared host parenchyma. A similar pattern of growth/sparing was also observed for TH-positive (noradrenergic) and SERT-positive (serotonergic) axons in the Fibro group (Fig. $7 D, D^{\prime}$, respectively).

$\leftarrow$

(Figure legand continues.) [SKP-SC]) or midlesion bridges (arrowheads in $\mathbf{G}, \boldsymbol{H}, \mathbf{G}^{\prime}, \boldsymbol{H}^{\prime}$ ) in the $S C$ groups. In the latter, axons were found in intact GFAP ${ }^{+}$regions or regions occupied by GFP ${ }^{+}$ SCS (arrowheads in $\boldsymbol{F}^{\prime}$ [higher magnification image of the box in $\boldsymbol{E}^{\prime}$ ]). The density of $\mathrm{TH}^{+}$and $\mathrm{SERT}^{+}$axons was highest rostral to the lesion and lowest caudal to the lesion in both SKP-SC ( $G$, $\left.\boldsymbol{G}^{\prime}\right)$ and N-SC $\left(\boldsymbol{E}, \boldsymbol{E}^{\prime}\right)$ groups and at the caudal extent of the lesion $\mathrm{TH}^{+}$and $\mathrm{SERT}^{+}$axons appeared to exit the spared rim (arrowheads in $\boldsymbol{F}, \boldsymbol{I}, \boldsymbol{I}^{\prime}$ [higher-magnification images of the boxes in $\left.\boldsymbol{E}, \boldsymbol{H}, \boldsymbol{H}^{\prime}\right]$ ) and the midlesion bridges (astrisks in $\boldsymbol{G}, \boldsymbol{H}, \boldsymbol{G}^{\prime}, \boldsymbol{H}^{\prime}$ ). All data are from longitudinal tissue sections at 11 wpi.
These axon populations primarily arise from the brainstem (locus ceruleus and raphe nuclei, respectively) in the uninjured spinal cord (Ramón-Cueto et al., 1998; Greene, 2006), so some portion of these fibers likely represent descending supraspinal axons. These subpopulations of putative brainstem-spinal axons were both found in very high density on the rostral side of the lesion in all cell transplant groups (TH: Fig. $7 D, E, G, H$; SERT: Fig. $\left.7 D^{\prime}, E^{\prime}, G^{\prime}, H^{\prime}\right)$. They were also found throughout the spared tissue rims and lesion-spanning bridges in both N-SC (TH: Fig. 7E; SERT: Fig. $7 E^{\prime}, F^{\prime}$; only spared rim depicted) and SKP-SC (TH: Fig. $7 G, H$; SERT: Fig. $7 G^{\prime}, H^{\prime}$; both spared rim and midlesion bridge depicted)-transplanted spinal cords, where there was a tendency for $\mathrm{TH}^{+}$axons to be denser in the midlesion bridges and SERT ${ }^{+}$axons to be denser in the spared rim (cf. Fig. $7 \mathrm{H}, \mathrm{H}^{\prime}$ ). The density of both $\mathrm{TH}^{+}$and SERT ${ }^{+}$axons declined near the caudal portion of the spared rim and tissue bridges, but a small number of fibers were observed on the caudal side of the lesion in all N-SC (TH: arrowheads in Fig. 7F; SERT: data not shown) and SKP-SC (TH: asterisks in Fig. 7G,H, arrowheads in Fig. 7I; SERT: asterisks in Fig. $7 G^{\prime}, H^{\prime}$, arrowheads in Fig. $7 I^{\prime}$ )-treated spinal cords. Therefore, the overall pattern of $\mathrm{TH}^{+}$and SERT ${ }^{+}$axon profiles was suggestive of descending, possibly supraspinal, axon growth through the spared rim and midlesion bridges.

In support of the notion that the size of this substrate somehow contributes to functional outcomes, we found a significant positive correlation between intact rim width and the intensity difference between the left and right forelimbs on the CatWalk in Experiment 2 (Spearman's $\rho=0.523, p=0.013$ ). This correlation indicates that animals with larger spared rim widths distributed their weight more evenly on the forelimbs after injury/treatment. Importantly, this correlation was significantly stronger if we excluded the animals in the Fibro group, many of which had a spared rim width of zero (Spearman's $\rho=0.731, p=0.005$ ), indicating that, even among SC-treated animals alone, more tissue sparing was associated with improved forelimb function.

\section{Discussion}

Here, we conducted two experiments using the DLF crush model of injury to examine the efficacy of neonatal SKP-SCs as an acute cellular therapy for incomplete cervical SCI in the rat. Compared with media treatment (Experiment 1), the transplantation of SKP-SCs was associated with increased use of the forelimb (left) affected by injury, enhanced RST axon densities rostral to the lesion, and red nucleus-evoked EMG motor thresholds and latencies closer to those of uninjured animals. Compared with Fibros (Experiment 2), the transplantation of neonatal SKP-SCs or $\mathrm{N}$-SCs improved weight distribution onto the left forelimb, enhanced sparing of the host tissue rim, and elevated RST axon counts at $\mathrm{C} 2$ and densities in the GM rostral to injury.

The mechanisms underlying the functional effects observed in Experiment 1 remain unclear because, although the transplantation of SKP-SCs was associated with enhanced RST axon density, this effect was only significant in the absence of CsA immunosuppression and did not correlate with behavioral or electrophysiological outcomes. Conversely, the reparative effects observed in Experiment 2 all appeared to be functionally relevant because increased spared rim width, elevated RST axon counts, and greater RST axon density rostral/caudal to injury were all correlated with improved behavioral or electrophysiological outcomes. The lack of correlations between RST axon measures and rearing behavior in either experiment suggests that plasticity of the injured RST may not play a major role in the recovery of overall forelimb use after DLF crush. In contrast, the correlations 
between RST axon measures and both the CatWalk and electrophysiological data in Experiment 2 suggest that new connections were formed between the injured RST and its original spinal targets. Given the lack of direct RST regeneration observed here, these new connections would have to be indirect, likely involving spared/sprouting propriospinal circuits that bypass the lesion site in the GM, similar to those reported previously after CST injury in the rat (Fouad et al., 2001; Bareyre et al., 2004). In addition, spared descending supraspinal motor tracts that were not traced in the present work, such as the CST or the contralateral RST, may have contributed to functional outcomes. Last, new connections may have occurred through the spared rim and midlesion bridges, as suggested by the serotonergic and noradrenergic axons observed crossing these substrates in SC-transplanted animals and the positive correlation between spared rim width and behavioral outcomes.

The distance between the transplanted cells and the injured RST fibers that responded to treatment here suggests that SCs exerted their influence via diffusible signals such as neurotrophic factors. Both N-SCs and SKP-SCs produce a variety of such factors (Reynolds and Woolf, 1993; Höke et al., 2006; Krause et al., 2014) and RST neurons are known to respond to some of these molecules (e.g., BDNF, NT-4/5, GDNF; Kobayashi et al., 1997; Dolbeare and Houle, 2003; Kwon et al., 2007). The elevation of neurotrophic factors is also known to enhance the excitability of uninjured circuits (Shu and Mendell, 1999; Arvanian and Mendell, 2001), which provides another means by which transplanted cells might contribute to functional outcomes regardless of any role they may play in promoting RST plasticity. Therefore, trophic effects may explain, at least in part, the functional advantages of SKP-SCs over media treatment in Experiment 1.

Although both SKP-SCs and N-SCs demonstrated therapeutic benefit after acute transplantation into the crushed DLF, neither cell type elicited regeneration of the injured RST. Furthermore, side-by-side testing of SKP-SC and N-SC transplants revealed no significant differences between these two groups on a wide variety of measures, including motor and electrophysiological functions, graft survival, neuroprotection, myelination, endogenous SC and astrocyte responses, migration/ integration into host parenchyma, and assessments of axonal sparing/sprouting. Our previous findings (Biernaskie et al., 2007) suggested that mouse neonatal SKP-SCs may have advantages over the adult N-SCs transplanted by other groups (Bunge and Wood, 2012) in terms of their interactions with astrocytes, their ability to migrate and integrate into spared host tissue, and their capacity to support the growth of descending brainstem axons. These advantages may reflect differences in the maturity of the cell types being compared (Fortun et al., 2009) or the species origin (mouse vs. rat) and our results here support that idea because we found no such differences between SKP-SCs and $\mathrm{N}-\mathrm{SCs}$ when both cell types were generated from neonatal rat tissue sources. Future efforts should be directed toward comparing neonatal versus adult SKP-SCs and N-SCs to determine whether any of these cell types are preferable as a therapy for SCI.

In Experiment 2, fibroblast transplantation was associated with decreased RST axon counts in the WM at C2. That finding could not be explained by group differences in BDA tracing or RST axon dieback, the latter of which does not reach C2 in the DLF crush injury model (Bretzner et al., 2008). Given that the transport of BDA is likely to fail in atrophied axons after SCI (Lasiene et al., 2008; W. Tetzlaff, unpublished observations), we surmise that the decline in BDA-labeled RST axons after fibroblast treatment here reflects decreased BDA transport due to more pronounced RST atrophy. Curiously, there were no signs of increased RST atrophy in the media-treated rats, which suggests that transplanted fibroblasts could have actually induced atrophy rather than merely failing to prevent it. This phenomenon may have resulted from ongoing compression of the spinal cord because we noted a buildup of connective tissue in the spinal canals of all fibroblast-treated rats, which might also partly explain the loss of intact host tissue (e.g., spared rim) observed in that group.

Graft volume for all SC-treated groups in the present study was $\sim 50 \%$ of what we anticipated based on our previous observations of SKP-SCs transplanted 1 week after thoracic contusion (Biernaskie et al., 2007). The lack of robust graft survival represents an important caveat to all of our findings because the results may have differed substantially if more cells had survived long term. The fact that CsA administration failed to improve graft volume indicates that $\mathrm{T}$-cell-mediated rejection did not play an extensive role in the loss of transplanted cells. We have observed that the oral delivery of CsA (Neoral) is as effective as daily intraperitoneal injections of CsA for promoting long-term survival of SKP-SCs after delayed (1-8 wpi) transplantation into thoracic contusions (J.S.S., P.A., and W.T., unpublished observations). Therefore, it seems unlikely that our findings were due to inadequate CsA levels and the acutely crushed cervical DLF may simply represent a harsher environment for transplanted SCs than subacute or even chronic thoracic contusion sites.

Regardless of graft survival, CsA had other interesting effects here because immunosuppression effectively eliminated group differences between media-treated and SKP-SC-treated rats on measures of RST sprouting/sparing in the GM rostral to injury. This finding was unexpected because neuroimmunophilin ligand immunosuppressants such as CsA and FK-506 have previously been reported to enhance axonal growth after SCI and nerve injury (Sugawara et al., 1999; Hayashi et al., 2005; Ibarra et al., 2007). However, it is important to note that CsA did not significantly alter RST densities in either of these groups, it merely caused slightly decreased RST densities in the SKP-SC group and slightly increased RST densities in the media group, and the combination of these nonsignificant effects resulted in the loss of significant group differences. These results suggest that CsA is not an ideal immunosuppressant drug for acute, nonautologous SC therapy after SCI, particularly in preclinical studies that assess axonal growth in response to these cells versus media treatment. Whether similar effects occur for other immunosuppressant drugs (e.g., FK-506), other cellular transplants, or delayed transplantation paradigms remain open questions for future research.

Here, for the first time, we demonstrated the efficacy of neonatal SKP-SCs as a treatment for cervical SCI. Hand/arm function is considered a high priority by individuals living with cervical SCI (Anderson, 2004), who account for $\sim 60 \%$ of the SCI population (National Spinal Cord Injury Statistical Center, 2014). Although the functional gains resulting from SC transplantation here were rather modest, they are very encouraging because even a small gain in hand/arm function can profoundly improve the performance of routine activities and quality of life after cervical SCI. Whereas a clinical intervention within minutes of injury would be impossible to achieve in traumatic SCI patients, our use of acute transplantation provides proof-ofprinciple that early intervention with SKP-SCs or N-SCs is beneficial after SCI. Our findings represent a promising beginning for the study of SKP-SCs as a treatment for cervical SCI, which should be expanded upon using cervical contusion models and feasibly delayed transplantation to maximize clinical rele- 
vance. Although there are potential sources of neonatal SKPs that could be used to generate SCs for clinical application, adult autologous skin is by far the most readily available source of SKPSCs for therapeutic application after SCI. Therefore, future work should focus on adult SKP-SCs, particularly SKP-SCs from adult human skin, because the latter represent the cells most likely to be applied in any clinical trials of SKP-SCs as a therapy for SCI.

\section{References}

Anderson KD (2004) Targeting recovery: priorities of the spinal cordinjured population. J Neurotrauma 21:1371-1383. CrossRef Medline

Arvanian VL, Mendell LM (2001) Acute modulation of synaptic transmission to motoneurons by BDNF in the neonatal rat spinal cord. Eur J Neurosci 14:1800-1808. CrossRef Medline

Bareyre FM, Kerschensteiner M, Raineteau O, Mettenleiter TC, Weinmann O, Schwab ME (2004) The injured spinal cord spontaneously forms a new intraspinal circuit in adult rats. Nat Neurosci 7:269-277. CrossRef Medline

Biernaskie JA, McKenzie IA, Toma JG, Miller FD (2006) Isolation of skinderived precursors (SKPs) and differentiation and enrichment of their Schwann cell progeny. Nat Protoc 1:2803-2812. Medline

Biernaskie J, Sparling JS, Liu J, Shannon CP, Plemel JR, Xie Y, Miller FD, Tetzlaff W (2007) Skin-derived precursors generate myelinating Schwann cells that promote remyelination and functional recovery after contusion spinal cord injury. J Neurosci 27:9545-9559. CrossRef Medline

Bretzner F, Arisato H, Biernaskie J, Liu J, Plunet W, Xu E, Borisoff J, McPhail L, Ramer M, Miller F, Tetzlaff W (2007) Skin-derived progenitors differentiated into Schwann cells (SKP-SC) promote motor plasticity after transplantation into the injured rat spinal cord. Soc Neurosci Abstr 33: 601.1.

Bretzner F, Liu J, Currie E, Roskams AJ, Tetzlaff W (2008) Undesired effects of a combinatorial treatment for spinal cord injury-transplantation of olfactory ensheathing cells and BDNF infusion to the red nucleus. Eur J Neurosci 28:1795-1807. CrossRef Medline

Bretzner F, Plemel JR, Liu J, Richter M, Roskams AJ, Tetzlaff W (2010) Combination of olfactory ensheathing cells with local versus systemic cAMP treatment after a cervical rubrospinal tract injury. J Neurosci Res 88:2833-2846. Medline

Bunge MB, Wood PM (2012) Realizing the maximum potential of Schwann cells to promote recovery from spinal cord injury. Handb Clin Neurol 109:523-540. CrossRef Medline

Dolbeare D, Houle JD (2003) Restriction of axonal retraction and promotion of axonal regeneration by chronically injured neurons after intraspinal treatment with glial cell line-derived neurotrophic factor (GDNF). J Neurotrauma 20:1251-1261. CrossRef Medline

Fortun J, Hill CE, Bunge MB (2009) Combinatorial strategies with Schwann cell transplantation to improve repair of injured spinal cord. Neurosci Lett 456:124-132. CrossRef Medline

Fouad K, Pedersen V, Schwab ME, Brösamle C (2001) Cervical sprouting of corticospinal fibers after thoracic spinal cord injury accompanies shifts in evoked motor responses. Curr Biol 11:1766-1770. CrossRef Medline

Greene JG (2006) Gene expression profiles of brain dopamine neurons and relevance to neuropsychiatric disease. J Physiol 575:411-416. CrossRef Medline

Hamers FP, Lankhorst AJ, van Laar TJ, Veldhuis WB, Gispen WH (2001) Automated quantitative gait analysis during overground locomotion in the rat: its application to spinal cord contusion and transection injuries. J Neurotrauma 18:187-201. CrossRef Medline

Hamers FP, Koopmans GC, Joosten EA (2006) CatWalk-assisted gait analysis in the assessment of spinal cord injury. J Neurotrauma 23:537-548. CrossRef Medline

Hayashi Y, Shumsky JS, Connors T, Otsuka T, Fischer I, Tessler A, Murray M (2005) Immunosuppression with either cyclosporine A or FK506 supports survival of transplanted fibroblasts and promotes growth of host axons into the transplant after spinal cord injury. J Neurotrauma 22: 1267-1281. CrossRef Medline

Hill CE, Moon LD, Wood PM, Bunge MB (2006) Labeled Schwann cell transplantation: cell loss, host Schwann cell replacement, and strategies to enhance survival. Glia 53:338-343. CrossRef Medline

Hilton BJ, Assinck P, Duncan GJ, Lu D, Lo S, TetzlaffW (2013) Dorsolateral funiculus lesioning of the mouse cervical spinal cord at $\mathrm{C} 4$ but not at $\mathrm{C} 6$ results in sustained forelimb motor deficits. J Neurotrauma 30:10701083. CrossRef Medline

Höke A, Redett R, Hameed H, Jari R, Zhou C, Li ZB, Griffin JW, Brushart TM (2006) Schwann cells express motor and sensory phenotypes that regulate axon regeneration. J Neurosci 26:9646-9655. CrossRef Medline

Hood B, Levene HB, Levi AD (2009) Transplantation of autologous Schwann cells for the repair of segmental peripheral nerve defects. Neurosurg Focus 26:e4. CrossRef Medline

Ibarra A, Hernández E, Lomeli J, Pineda D, Buenrostro M, Martiñón S, Garcia E, Flores N, Guizar-Sahagun G, Correa D, Madrazo I (2007) Cyclosporin-A enhances non-functional axonal growing after complete spinal cord transection. Brain Res 1149:200-209. CrossRef Medline

Kobayashi NR, Fan DP, Giehl KM, Bedard AM, Wiegand SJ, Tetzlaff W (1997) BDNF and NT-4/5 prevent atrophy of rat rubrospinal neurons after cervical axotomy, stimulate GAP-43 and Talpha1-tubulin mRNA expression, and promote axonal regeneration. J Neurosci 17:9583-9595. Medline

Krause MP, Dworski S, Feinberg K, Jones K, Johnston APW, Paul S, Paris M, Peles E, Bagli D, Forrest CR, Kaplan DR, Miller FD (2014) Direct genesis of functional rodent and human Schwann cells from skin mesenchymal precursors. Stem Cell Reports 3:85-100. CrossRef Medline

Küchler M, Fouad K, Weinmann O, Schwab ME, Raineteau O (2002) Red nucleus projections to distinct motor neuron pools in the rat spinal cord. J Comp Neurol 448:349-359. CrossRef Medline

Kwon BK, Liu J, Lam C, Plunet W, Oschipok LW, Hauswirth W, Di Polo A, Blesch A, Tetzlaff W (2007) Brain-derived neurotrophic factor gene transfer with adeno-associated viral and lentiviral vectors prevents rubrospinal neuronal atrophy and stimulates regeneration-associated gene expression after acute cervical spinal cord injury. Spine 32:1164-1173. CrossRef Medline

Lasiene J, Shupe L, Perlmutter S, Horner P (2008) No evidence for chronic demyelination in spared axons after spinal cord injury in a mouse. J Neurosci 28:3887-3896. CrossRef Medline

Li WW, Penderis J, Zhao C, Schumacher M, Franklin RJ (2006) Females remyelinate more efficiently than males following demyelination in the aged but not young adult CNS. Exp Neurol 202:250-254. CrossRef Medline

Liu Y, Kim D, Himes BT, Chow SY, Schallert T, Murray M, Tessler A, Fischer I (1999) Transplants of fibroblasts genetically modified to express BDNF promote regeneration of adult rat rubrospinal axons and recovery of forelimb function. J Neurosci 19:4370-4387. Medline

Muir GD, Whishaw IQ (2000) Red nucleus lesions impair overground locomotion in rats: a kinetic analysis. Eur J Neurosci 12:1113-1122. CrossRef Medline

National Spinal Cord Injury Statistical Center, Birmingham, AL (2014) Spinal cord injury facts and figures at a glance. https://www.nscisc.uab.edu.

Pearse DD, Marcillo AE, Oudega M, Lynch MP, Wood PM, Bunge MB (2004) Transplantation of Schwann cells and olfactory ensheathing glia after spinal cord injury: does pretreatment with methylprednisolone and interleukin-10 enhance recovery? J Neurotrauma 21: 1223-1239. Medline

Ramer LM, Borisoff JF, Ramer MS (2004a) Rho-kinase inhibition enhances axonal plasticity and attenuates cold hyperalgesia after dorsal rhizotomy. J Neurosci 24:10796-10805. CrossRef Medline

Ramer LM, Au E, Richter MW, Liu J, Tetzlaff W, Roskams AJ (2004b) Peripheral olfactory ensheathing cells reduce scar and cavity formation and promote regeneration after spinal cord injury. J Comp Neurol 473:1-15. CrossRef Medline

Ramón-Cueto A, Plant GW, Avila J, Bunge MB (1998) Long-distance axonal regeneration in the transected adult rat spinal cord is promoted by olfactory ensheathing glia transplants. J Neurosci 18:3803-3815. Medline

Reynolds ML, Woolf CJ (1993) Reciprocal Schwann cell-axon interactions. Curr Opin Neurobiol 3:683-693. CrossRef Medline

Richter MW, Fletcher PA, Liu J, Tetzlaff W, Roskams AJ (2005) Lamina propria and olfactory bulb ensheathing cells exhibit differential integration and migration and promote differential axon sprouting in the lesioned spinal cord. J Neurosci 25:10700-10711. CrossRef Medline

Schallert T, Fleming SM, Leasure JL, Tillerson JL, Bland ST (2000) CNS plasticity and assessment of forelimb sensorimotor outcome in unilateral 
rat models of stroke, cortical ablation, parkinsonism and spinal cord injury. Neuropharmacology 39:777-787. CrossRef Medline

Shu XQ, Mendell LM (1999) Neurotrophins and hyperalgesia. Proc Natl Acad Sci U S A 96:7693-7696. CrossRef Medline

Sparling JS, Bretzner F, Biernaskie J, Jiang Y, Lucero-Villegas F, Assinck A, Morano T, Liu J, Miller FD, Tetzlaff W (2009) Schwann cells generated from skin-derived precursors show discrete advantages over those harvested from peripheral nerve after transplantation into incomplete cervical spinal cord injury. 13th International Symposium on Neural Regeneration, Pine Grove, CA. Neurorehabil Neural Repair 23:954-1000. CrossRef

Sugawara T, Itoh Y, Mizoi K (1999) Immunosuppressants promote adult dorsal root regeneration into the spinal cord. Neuroreport 10:3949-3953. CrossRef Medline

Tetzlaff W, Okon EB, Karimi-Abdolrezaee S, Hill CE, Sparling JS, Plemel JR, Plunet WT, Tsai EC, Baptiste D, Smithson LJ, Kawaja MD, Fehlings MG, Kwon BK (2011) A systematic review of cellular transplantation thera- pies for spinal cord injury. J Neurotrauma 28:1611-1682. CrossRef Medline

Toma JG, Akhavan M, Fernandes KJ, Barnabé-Heider F, Sadikot A, Kaplan DR, Miller FD (2001) Isolation of multipotent adult stem cells from the dermis of mammalian skin. Nat Cell Biol 3:778-784. CrossRef Medline

Vrinten DH, Hamers FF (2003) 'CatWalk' automated quantitative gait analysis as a novel method to assess mechanical allodynia in the rat: a comparison with von Frey testing. Pain 102:203-209. CrossRef Medline

Vroemen M, Caioni M, Bogdahn U, Weidner N (2007) Failure of Schwann cells as supporting cells for adult neural progenitor cell grafts in the acutely injured spinal cord. Cell Tissue Res 327:1-13. Medline

Wyndaele M, Wyndaele JJ (2006) Incidence, prevalence and epidemiology of spinal cord injury: what learns a worldwide literature survey? Spinal Cord 44:523-529. CrossRef Medline 